\title{
WestVirginiaUniversity
}

THE RESEARCH REPOSITORY @ WVU

Graduate Theses, Dissertations, and Problem Reports

2008

\section{Relay communications over frequency-selective fading channels}

John Paul Mazzie

West Virginia University

Follow this and additional works at: https://researchrepository.wvu.edu/etd

\section{Recommended Citation}

Mazzie, John Paul, "Relay communications over frequency-selective fading channels" (2008). Graduate Theses, Dissertations, and Problem Reports. 1936.

https://researchrepository.wvu.edu/etd/1936

This Thesis is protected by copyright and/or related rights. It has been brought to you by the The Research Repository @ WVU with permission from the rights-holder(s). You are free to use this Thesis in any way that is permitted by the copyright and related rights legislation that applies to your use. For other uses you must obtain permission from the rights-holder(s) directly, unless additional rights are indicated by a Creative Commons license in the record and/ or on the work itself. This Thesis has been accepted for inclusion in WVU Graduate Theses, Dissertations, and Problem Reports collection by an authorized administrator of The Research Repository @ WVU. For more information, please contact researchrepository@mail.wvu.edu. 


\title{
Relay Communications Over Frequency-Selective Fading Channels
}

\author{
by \\ John Paul Mazzie \\ Thesis submitted to the \\ College of Engineering and Mineral Resources \\ at West Virginia University \\ in partial fulfillment of the requirements \\ for the degree of \\ Master of Science \\ in \\ Electrical Engineering \\ Daryl Reynolds, Ph.D. \\ Natalia Schmid, Ph.D. \\ Matthew C. Valenti, Ph.D., Chair \\ Lane Department of Computer Science and Electrical Engineering \\ Morgantown, West Virginia \\ 2008
}

Keywords: Frequency-Selective Fading, Relay, Outage Probability, HSDPA

Copyright 2008 John Paul Mazzie 


\author{
Abstract \\ Relay Communications Over Frequency-Selective Fading Channels \\ by \\ John Paul Mazzie \\ Master of Science in Electrical Engineering \\ West Virginia University \\ Matthew C. Valenti, Ph.D., Chair
}

Wireless communications over long distances can be assisted by a third radio acting as a relay. If the relay is placed close to the source, then the source-relay link will be characterized as a fairly benign additive white Gaussian noise (AWGN) channel. However, the long distance link from relay to destination is susceptible to frequency-selective fading. This thesis explores the design and analysis of a particular relay communication system characterized by a low power source, a relay that is close to the source, and a frequencyselective channel from relay to destination. Because the direct link from source to destination is very weak, it is not exploited, but rather communications is via a traditional two-hop process.

Link design is based on the high speed download packet access (HSDPA) standard, which uses a combination of turbo coding, hybrid-ARQ, and multicode CDMA. To provide further diversity, the relay-destination link uses a secondary spreading code, rake reception, and multiple receive antennas. An extensive analysis was conducted to study the influence of a wide variety of link configurations and channel conditions. The study was accelerated through the use of a quasi-analytical approach based on the concept of information-outage, which allows the link to be simulated without requiring a turbo decoder. 


\section{Acknowledgements}

I would first like to thank Dr. Valenti, who has been of great help in completing this thesis. There is no way I could have made it through without his guidance. I would also like to thank the members of my committee, Dr. Reynolds and Dr. Schmid. I have enjoyed taking their classes, along with the challenges they presented.

I would like to also thank my friends, particularly David Buckingham, Phillip Green, and Josh Pulice. They made this extra time in school much more bearable. Without them, I am not sure I would have had the energy to continue my studies. Lastly, I would like to thank my parents who have supported everything that I have done throughout my life, and will continue to do so. 


\section{Contents}

$\begin{array}{ll}\text { Acknowledgements } & \text { iii }\end{array}$

List of Figures $\quad$ vi

List of Tables viii

Notation $\quad$ ix

1 Introduction $\quad 1$

1.1 Introduction . . . . . . . . . . . . . . . . . . 1

1.2 Thesis Outline . . . . . . . . . . . . . . . . . 3

2 System and Channel Model 6

2.1 Signal-Space Model of Digital Communications . . . . . . . . . . . . . 6

2.1.1 Communication System Model . . . . . . . . . . . . . . . 6

2.1.2 Representation of Digital Signals . . . . . . . . . . . . . . . . . 8

2.2 Fading Channel . . . . . . . . . . . . . . . . . . . . . . . 9

2.2.1 Flat Fading . . . . . . . . . . . . . . . . . . . . . . . . . . . . . . . . . . . 10

2.2.2 Frequency-Selective Fading . . . . . . . . . . . . . . . 11

2.3 Channel Simulator . . . . . . . . . . . . . . . . . . . 11

2.3.1 Implementation . . . . . . . . . . . . . . . . . . . . 11

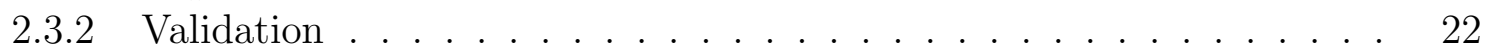

3 Source to Relay Link $\quad 26$

3.1 HSDPA Overview . . . . . . . . . . . . . . . . . 26

3.2 Source to Relay Link . . . . . . . . . . . . . . . . . . . . . . . . . . . . . . . . . . . . . . .

3.3 Image Processing . . . . . . . . . . . . . . . . . . . . . 37

4 Information-Outage Analysis $\quad 39$

4.1 Information-Outage . . . . . . . . . . . . . . . . . . . . . 39

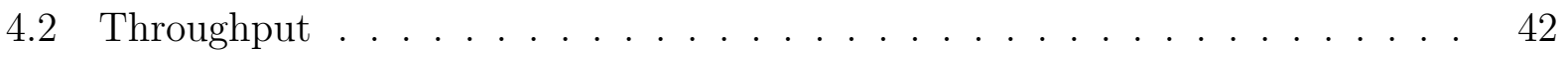

5 Relay to Destination Link $\quad 45$

5.1 Modulation and Coding Schemes . . . . . . . . . . . . . . . 45

5.2 Link Analysis . . . . . . . . . . . . . . . . . . . . . . 48 
6 Conclusions and Future Work $\quad 56$

6.1 Conclusions . . . . . . . . . . . . . . . . . . 56

6.2 Suggestions for Future Work . . . . . . . . . . . . . . . 57

$\begin{array}{ll}\text { References } & 59\end{array}$ 


\section{List of Figures}

1.1 Diagram of Relay System . . . . . . . . . . . . . . . . . . . . . 2

2.1 Communications System Model . . . . . . . . . . . . . . . . . . . . 6

2.2 QPSK Signal Space Representation . . . . . . . . . . . . . . . . . . . 9 9

2.3 Model of Flat Fading Channel . . . . . . . . . . . . . . . . . . . . . . . 9

2.4 [1] Frequency-Selective Bandwidth Comparison . . . . . . . . . . . . . . 12

2.5 Rayleigh Flat Fading Simulator $\left(f^{-4}\right) \ldots \ldots \ldots$

2.6 Rician Flat Fading Simulator $\left(f^{-4}\right) \ldots \ldots \ldots \ldots$

2.7 Power Amplitude of Rayleigh Fading Channel $\left(S_{4}=1, f^{-4}\right.$, and $\left.\tau_{0} / T_{s}=100\right) \quad 17$

2.8 Phase of Rayleigh Fading Channel $\left(S_{4}=1, f^{-4}\right.$, and $\left.\tau_{0} / T_{s}=100\right) \ldots 17$

2.9 Cumulative Distribution Function of Rician Fading, $f^{-4}$ Doppler Spectrum,

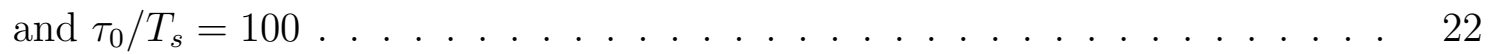

2.10 Mean Up Level Crossing Rate of Rician Fading, $f^{-4}$ Doppler Spectrum, and

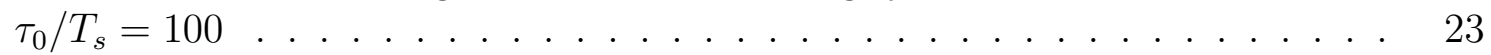

2.11 Mean Fade Duration of Rician Fading, $f^{-4}$ Doppler Spectrum, and $\tau_{0} / T_{s}=10023$

3.1 Baseband processing at HSDPA transmitter . . . . . . . . . . . . 27

3.2 UMTS Turbo Encoder . . . . . . . . . . . . . . . . . . . . . . . . . . 28

3.3 16-QAM Signal Constellation . . . . . . . . . . . . . . . . . . 30

3.4 H-Set 6 HARQ Interleaving with 6 processes . . . . . . . . . . . . . 31

3.5256 by 256 grey-scale image using 4 bit quantization . . . . . . . . . . . 32

3.6 Bit Error Performance of two QPSK-based systems in AWGN . . . . . . . . 36

3.7 Bit Error Performance of one QPSK-based and two QAM-based systems in AWGN . . . . . . . . . . . . . . . . . . . . 36

3.8 Comparison of received images at different error rates . . . . . . . . . . . 37

4.1 Information-Outage Probability Concept . . . . . . . . . . . . . . . 40

4.2 Comparison of IOP and FER over three AWGN Channels . . . . . . . . . 42

4.3 Comparison of simulation runtime . . . . . . . . . . . . . 43

5.1 Frame Error Rate and Information-Outage Probability Rate for MCS 1 . . 49

5.2 Throughput of MCS $1 \ldots \ldots \ldots 4 . \ldots \ldots$

5.3 Influence of the amount of rake fingers and number of antennas on MCS 1 . 51

5.4 Throughput of all MCS schemes over the a fading channel . . . . . . . . 51

5.5 Influence of Signal Decorrelation Time on System . . . . . . . . . . . . 52 
5.6 Influence of Frequency-Selective Bandwidth on System . . . . . . . . . . 53

5.7 Comparison of IOP and log-MAP Decoded system throughputs . . . . . . . 54

6.1 Histogram of Bit Errors for 1 million turbo codewords over AWGN channel . 57 


\section{List of Tables}

3.1 QPSK at $10 \mathrm{fps}$ Video Transmission Rate . . . . . . . . . . . . . . 33

3.2 QPSK at $20 \mathrm{fps}$ Video Transmission Rate . . . . . . . . . . . . . . 33

3.3 16-QAM at $20 \mathrm{fps}$ Video Transmission Rate . . . . . . . . . . . . . . . . 34

3.4 16-QAM at $28 \mathrm{fps}$ Video Transmission Rate . . . . . . . . . . . . . . . . . . 34

5.1 MCS 1 - H-Set 3 QPSK . . . . . . . . . . . . . 46

5.2 MCS 2 - H-Set 3 16-QAM . . . . . . . . . . . . . . 46

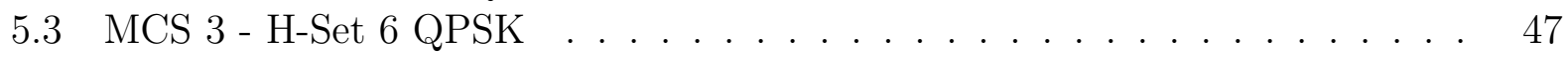

5.4 MCS 4 - H-Set 6 QAM . . . . . . . . . . . . . . 47 


\section{Notation}

We use the following notation and symbols throughout this thesis.

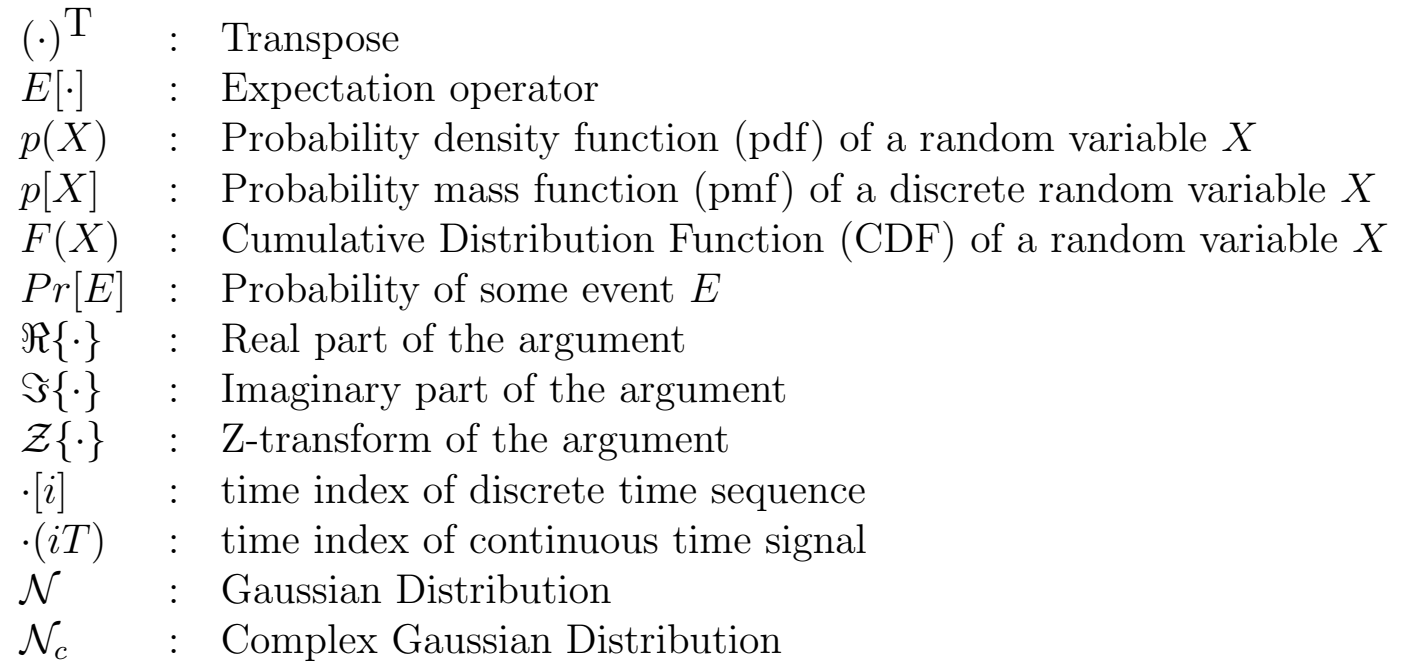

Bold lower case letters denote row vectors. 


\section{Chapter 1}

\section{Introduction}

\section{$1.1 \quad$ Introduction}

Wireless communications over long distances is difficult, but the addition of relays to the system can help to increase performance. Long distance links are likely to also likely to have a frequency-selective channel, on top of the free space losses. The system we are analyzing is a two-hop system involving a low power source, a relay close to the source, and a destination which is much farther away. Since the first link is so short, it will act like an additive white Gaussian noise channel, while the second, longer, link will be frequency-selective.

Figure 1.1 shows a relaying system, which contains three nodes. The first node is the source, which will produce information, for instance imagery or video, and transmit to the relay. The second node is the relay, which transmits the data received from the source and sends it to the third node, the destination. This system is optimized for the transmission of the images and video taken at the source. The signal sent from the source to relay is relatively weak, but since the distance between the source and the relay is so close, there is little attenuation so will be sufficient for reliable detection. The relay is equipped with a high power amplifier in order to transmit data to the destination at a higher power.

The source of this system has one antenna to transmit data with, which is pointed to the relay. The relay has a receive antenna aimed at the source, and another antenna pointed toward the destination. The relay node is close to the source while the destination is very far away from the source. The destination has multiple receive antennas (up to 3 for this 


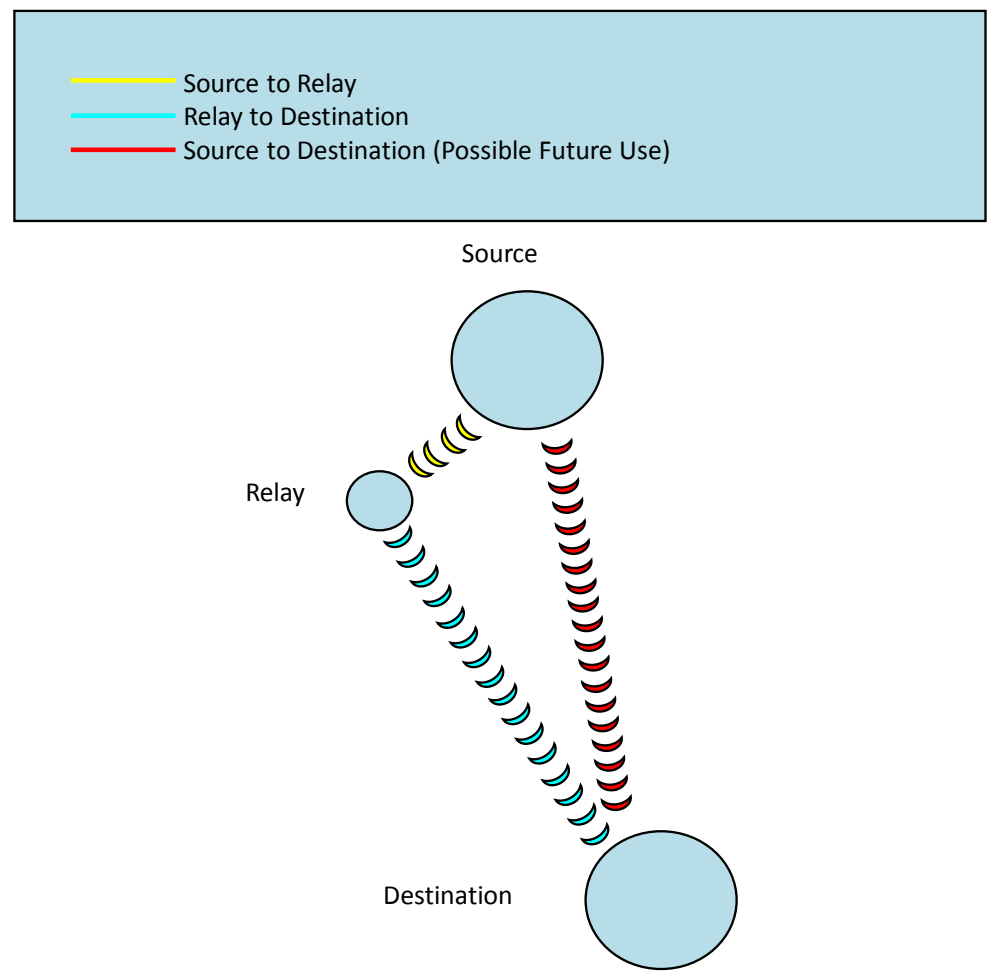

Figure 1.1: Diagram of Relay System

thesis), which could be spaced widely apart.

The source begins the process by transmitting uncompressed images over the high-speed short distance link to the relay. These transmissions are done using turbo coding, directsequence spread spectrum (DSSS), and two different modulation types (QPSK or 16-QAM), at three different throughput rates (2.7 Mbps, 5.4 Mbps, or 7.56 Mbps). The images (frames of video) are assumed to be 256 by 256 pixels in size, with 4 bits of resolution, and will be displayed in greyscale, at framerates of 10, 20, or 28 frames per second (fps).

After the initial transmission from the source is received at the relay, lossless image compression is performed. The compacted (lossless compression) data, which will vary in size, is sent over a link, which will be turbo coded, and use DSSS with either QPSK or 16-QAM modulation. The main difference between this link and the one from source to relay is that it will use hybrid-ARQ (HARQ). The reason that HARQ is used on the link from relay to destination, is due to the fact that it experiences fading. The performance will 
benefit from the added time diversity provided by using HARQ.

This works by the destination decoding each transmission from the relay, and checking the embedded cyclic redundancy check (CRC) code to determine if uncorrected errors occurred. If errors were detected, the destination will send a request to the relay for a retransmission. Because there could be data retransmissions and the frame size variability due to compaction, the frame rate will not be constant, like in the first link. In the analysis, discussed later in Section 5.2, the frame rate is determined as a function of signal-to-noise ratio for different channel conditions, link configurations, and receiver implementations.

Both links in this system use technology based on the high-speed downlink packet access (HSDPA) standard, which is used by the wireless cell phone industry to provide high speed download access to subscribers. By basing the system on HSDPA commercial standard, the concept is more likely to be used in the commercial world, and can use devices widely available from different vendors.

Capacity theorems for relay channels are presented in [2] and [3] considers coded systems performance over block fading channels. Also, [4] explores a block-OFDMA method for relay assisted transmission on frequency-selective fading channels. Cooperative diversity in wireless networks is demonstrated in [5] and [6] and Hybrid-ARQ over relaying channels is presented in [7].

\subsection{Thesis Outline}

The main contribution is the application of information-outage analysis to a two-hop network that accounts for frequency-selective fading and hybrid-ARQ signaling. This type of analysis is used to significantly increase the run-time of simulations for many different channel conditions and receiver configurations. The increase in speed in turn allows the selection of channel conditions and receiver configurations for use in turbo decoded simulations, which are much slower but give results of actual performance.

Chapter 2 contains a quick review of digital communications, and a description of the fading channel. Even though it is assumed that the short link from source to relay is an additive white Gaussian noise (AWGN) channel, this simplistic approach is not realistic for 
the longer link between relay and destination. A description of the implementation of the frequency-selective fading channel model (based on the models described in [8]) along with validation (based on [9]) is also included in this chapter.

While the links are both based on HSDPA, there is a problem with it for the longer link from relay to destination. The spreading codes that are used (Walsh Codes with a spreading factor of 16), do not have very good resistance to intersymbol interference (ISI) that occurs on frequency-selective channels. In order to counteract this, a couple of options are proposed. One is to use equalization at the receiver, but this would increase the complexity of the receiver, while not giving the frequency diversity provided by having a wider bandwidth. Another option is to increase the transmitted bandwidth, by using a secondary spreading code. The Barker code with a spreading factor of 11 was chosen to increase the RF bandwidth to around $50 \mathrm{MHz}$, and also because it has good autocorrelation properties. Since a Barker code is used, this allows the use of a rake receiver to provide more diversity at the destination. These cumulative effects are also discussed in Chapter 2.

The first section of Chapter 3 provides an overview of the HSDPA standard, while the successive sections talk about the link from source to relay and the image processing that occurs at the relay. The link from source to relay, including HSDPA parameters, are part of section 3.2. The bit error rate performance of this link over four different configurations, which support different video frame rates, are shown. A non-negligible bit error is assumed to exist on this link, and example images sent over this link that shows the impact of uncorrected errors on the image, and are shown.

Section 3.3 describes the image processing of the relay. Once the images are received at the relay from the source, compaction (lossless compression) will be performed on it before forwarding to the destination. The proposal for this compression is the Lempel-Ziv-Welch (LZW) algorithm for lossless compression. The compression is performed on each frame of video, without trying to exploit correlation between images.

Chapter 4 is devoted to the concept of using information-outage probability (IOP) to speed up simulation runtime. These simulation results will be used in order to provide link scheme and receiver designs to more accurately simulate using the frame error rate (FER) of the turbo code. The runtime of a full turbo-decoded system modulation is long, causing 
problems when trying to determine system models for use. The proposal of Section 4.1 is to remove the turbo decoder and generate information-outage curves, where the justification is that turbo codes are capacity approaching. Section 4.2 describes how the throughput of the system is determined using the frame error rate of the system or information outage probability curve.

The link from relay to destination is described in Chapter 5. This link, like that of source to relay, is also based on the HSDPA standard, but will operate in hybrid-ARQ mode. If the destination receives a codeword that it cannot decode, a retransmission will be requested. According to the standard, a maximum of four transmissions (including the initial) will be attempted. If all four attempts are made without success, the system will give up and move on to the next transmission. Extensive simulation results for the relay to destination link are shown, mostly using the IOP concept from Chapter 4.1. These results are in terms of information outage curves with comparison to the full turbo decoded system, as well as throughput for both types of simulations when hybrid-ARQ is used. The effects of having multiple antennas for the destination, as well as the number of fingers on the rake receivers, and the channel model parameters (signal decorrelation time $\tau_{0}$, and frequency-selective bandwidth $f_{0}$ ) are explored.

In Section 6.1, the results of the link analysis of Chapter 5 are summarized and conclusions are made. Also, in Section 6.2, possible ways to use the weak link from source to destination in order to improve performance are contemplated, though it appears too weak to exploit easily. 


\section{Chapter 2}

\section{System and Channel Model}

\subsection{Signal-Space Model of Digital Communications}

Innovations over the last several decades in hardware and digital signal processing have made digital communication systems cheaper, faster, and more power efficient than analog communication systems. Also digital modulation has many advantages to analog modulation, such as high spectral efficiency, powerful error correction, as well as improved security and privacy.

\subsubsection{Communication System Model}

Figure 2.1 shows the basic representation of a digital communications system. The input of the system could be analog (audio, video) or digital (morse code), with a finite number

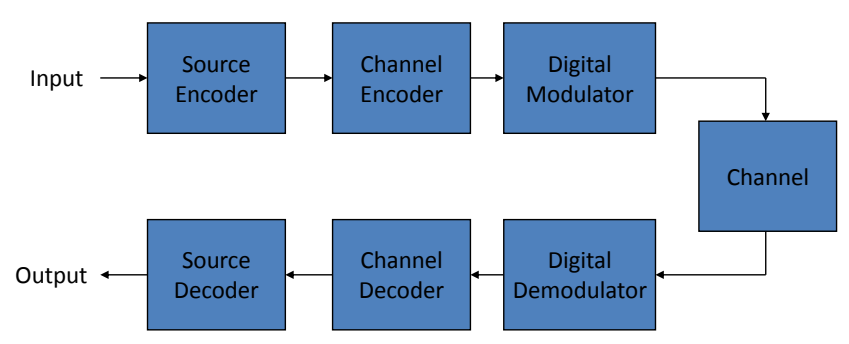

Figure 2.1: Communications System Model 
of output characters. In a digital communication system, the input message needs to be converted into binary digits (if necessary) and compressed. This representation of the input message $(\mathbf{m})$, which is the output of the source encoder, will result in little or no redundancy.

Once the output from the source encoder, called the information sequence, is obtained it is sent to the channel encoder. The channel encoder is used to introduce redundancy into the system, which will help the receiver reduce the effects of noise and interference introduced during transmission through the channel. This added redundancy increased the reliability of the received data, making the output more accurate. The encoder adds this redundancy, by taking the $k$ bits of data in the information sequence and converting it to an $n$ length codeword (c). The ratio of $k / n$ is called the code rate, and lets us know how efficient the code is.

Next, the codeword is sent to the digital modulator, which is the interface to the communication channel. The digital modulator's purpose is to map the codeword onto signal waveforms. A simple example would be a system that sends one bit of information at a time, where bit 1 would be mapped to waveform $s_{1}(t)$ and bit 0 is mapped to waveform $s_{0}(t)$. This simple modulation is called binary modulation, but more sophisticated mapping can be created, which are capable of sending a larger number of bits in each waveform. If the system would like to send $b$ coded bits at a time, $M=2^{b}$ distinct waveforms $s_{i}(t), i=0,1, \ldots, M-1$ are required to send that data. This is called $M$-ary modulation, where $M>2$.

From the digital modulator, the waveforms are sent over channel, which is a physical medium connecting the transmitter and receiver. When communications is wireless the medium is usually the atmosphere and a pair of antennas, while wired communications uses copper lines, fiber optics, or other physical media. The channel generally adds random artifacts to the signal being sent over them, which cause corruptions. There are many factors that can play into this corruption, such as thermal noise, lightning, or even other systems communicating over the same medium.

When the message is received, the digital demodulator takes the corrupted transmitted waveform $(r(t))$, and attempts to convert back to the $b$ coded bits that were input into the digital modulator. These coded bits $(\hat{\mathbf{c}})$ are then sent to the channel decoder, which then attempts to reconstruct the information sequence $(\hat{\mathbf{m}})$ from knowledge about the code used 
and the redundancy added by the channel encoder to the transmitted data.

After the information sequence $(\hat{\mathbf{m}})$ is obtained from the channel decoder, it is then sent to the source decoder. The source decoder is where the original source signal is reconstructed. This reconstruction is only an approximation of the original source, due to the distortions introduced by the source encoder and channel decoder. The differences between the original and the reconstruction are a measure of the distortion of the communication system.

The performance of the system is measured by how many errors occur in the decoded information sequence $(\hat{\mathbf{m}})$. The average probability of a bit error at the output of the decoder is actually the performance of the demodulator-decoder combination. In this way the probability of error is a function of the characteristics of the code, modulation type, transmit power, channel characteristics, and demodulation and decoding methods [10].

\subsubsection{Representation of Digital Signals}

The building block of representing digital signals is the basis. Using a Gram-Schmidt orthonormalization technique, any set of $M$ real signals $\mathcal{S}=\left\{s_{1}(t), s_{2}(t), \ldots, s_{M}(t)\right\}$ can be represented by a linear combination of $N \leq M$ orthonormal basis functions represented by $\left\{\phi_{1}(t), \phi_{2}(t), \ldots, \phi_{N}(t)\right\}$. These signals contained in the set $\mathcal{S}$ are defined over $[0, T)$ and have finite energy. The signal can now be expressed, $s_{i}(t) \in \mathcal{S}$, in terms of its basis function representation as

$$
s_{i}(t)=\sum_{i=1}^{N} s_{i j} \phi_{j}(t), 0 \leq t<T_{s}
$$

where

$$
s_{i j}=\int_{0}^{T} \phi_{i}(t) \phi_{j}(t) d t
$$

is a real valued coefficient that represents the projection of $s_{i}(t)$ on the basis function $\phi_{j}(t)$. The number of basis functions $N=M$ only when the signals $s_{i}(t)$ are orthogonal, otherwise $N<M$. [1]

Each signal can also be represented $s_{i}(t)$ using a signal vector $\mathbf{s}_{i}=\left[s_{i 1}, s_{i 2}, \ldots, s_{i N}\right]$. If the basis functions are also placed into a vector $\phi=\left[\phi_{1}(t), \phi_{2}(t), \ldots, \phi_{N}(t)\right]$, the original signal can be determined by

$$
s_{i}(t)=\mathbf{s}_{i} \cdot(\phi)^{\mathrm{T}}, 0 \leq t<T_{s}
$$




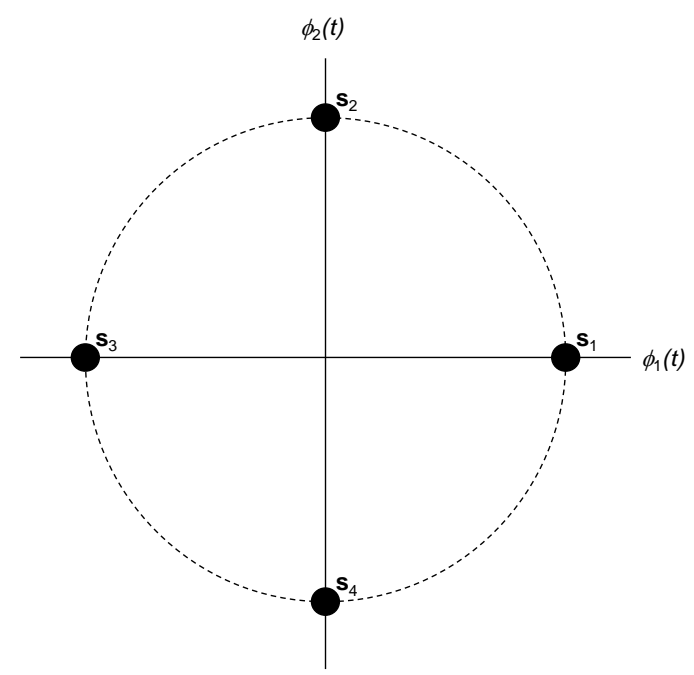

Figure 2.2: QPSK Signal Space Representation

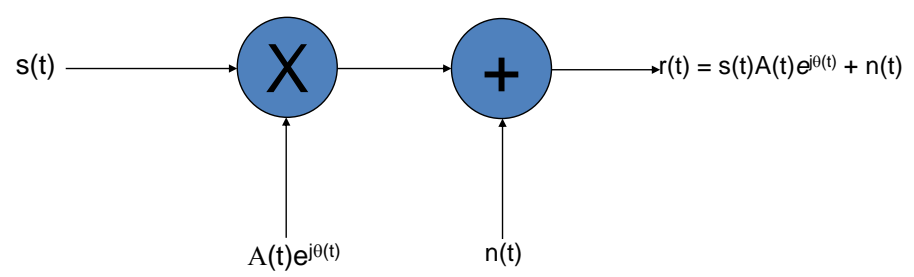

Figure 2.3: Model of Flat Fading Channel

where $(\phi)^{\mathrm{T}}$ denotes the transpose of $\phi$. An example using Quadrature Phase Shift Keying (QPSK) modulation, can be seen in Figure 2.2, where the signals can be represented as $\mathbf{s}_{1}=[1,0], \mathbf{s}_{2}=[0,1], \mathbf{s}_{3}=[-1,0]$, and $\mathbf{s}_{4}=[0,-1]$.

\subsection{Fading Channel}

When signals are passed over long distances, or along cluttered paths, such as mountainous or urban environments, signal fading often occurs. Figure 2.3 shows a model describing a flat fading channel where $A(t)$ is the amplitude fading coefficient, $\theta(t)$ is the phase of the 
fading channel, and $n(t)$ is additive white Gaussian noise (AWGN). In a system where only AWGN is involved, and there is no fading, the fading coefficient $A(t)$ would be set to 1 , and $\theta(t)$ to 0 . In a channel where flat fading is involved the fading coefficient would have its own statistical distribution, such as Rician or Rayleigh distributions. This fading is caused by multipath components of the signal which reach the receiver at different times, with different powers and phases. This can cause constructive interference as well as destructive interference, which will amplify the signal or attenuate the signal at the receiver, respectively [8].

\subsubsection{Flat Fading}

In flat fading the bandwidth of the signal modulation is small in comparison to the coherence bandwidth, also known as the frequency-selective bandwidth, of the channel $\left(f_{0}\right)$. This means that the attenuation of the channel is generally flat over the bandwidth of the signal. When this type of fading occurs the effect can be represented mathematically by multiplication of the transmitted signal and the fading factor of the communication channel. So, the real transmitted signal can be represented by

$$
s_{T}(t)=\Re\left\{s_{i}(t) e^{j \omega_{c} t}\right\}
$$

where $s_{i}(t)$ is the modulated symbol, and $\omega_{c}$ is the carrier angular frequency. The received signal of a flat fading channel in the absence of noise can then be expressed by

$$
s_{R}(t)=\Re\left\{A(t) e^{j \theta(t)} s_{i}(t) e^{j \omega_{c} t}\right\}
$$

where $A(t)$ is the time varying amplitude of the communication channel, and $\theta(t)$ is the phase of the channel. The in-phase $(I(t))$ and quadrature-phase $(Q(t))$ components of the channel gain are represented as

$$
\begin{aligned}
& I(t)=\Re\left\{A(t) e^{j \theta(t)}\right\}=A(t) \cos (\theta(t)) \\
& Q(t)=\Im\left\{A(t) e^{j \theta(t)}\right\}=A(t) \sin (\theta(t))
\end{aligned}
$$




\subsubsection{Frequency-Selective Fading}

Frequency-selective fading occurs when the bandwidth of the modulated signal is large in comparison to the coherence bandwidth of the channel. This channel cannot be represented by a multiplication factor on the transmitted signal, but must be represented by a timevarying linear filter. In this model, the received signal is expressed by the convolution of the complex envelope of the transmitted signal from Equation 2.4 and the channel impulse response function in absence of noise is shown as

$$
\begin{gathered}
s_{R}(t)=\Re\left\{r(t) e^{j \omega_{c} t}\right\} \\
r(t)=\int_{0}^{\infty} s_{i}(t-\tau) h(t, \tau) d \tau
\end{gathered}
$$

where $r(t)$ is the complex envelope of the received signal, and $h(t, \tau)$ is the time-varying impulse response of the communication channel.

The frequency-selective bandwidth $\left(f_{0}\right)$ is the main benchmark to determine how frequencyselective a channel is. The equation to determine $f_{0}$ is

$$
f_{0}=1 /\left(2 \pi \sigma_{t}\right)
$$

where $\sigma_{t}$ is the standard deviation of signal time delay jitter that comes from angular scattering. The value of $f_{0}$ shows what the maximum modulation rate that the channel supports negligible intersymbol interference (ISI). Small values of $f_{0}$ are indicative of sever frequencyselectivity, while large values correspond to flat fading. This comparison is shown in Figure 2.4 .

\subsection{Channel Simulator}

\subsubsection{Implementation}

The implementation of the following simulator is derived from the model of Bogusch [8] and Dana [9]. 


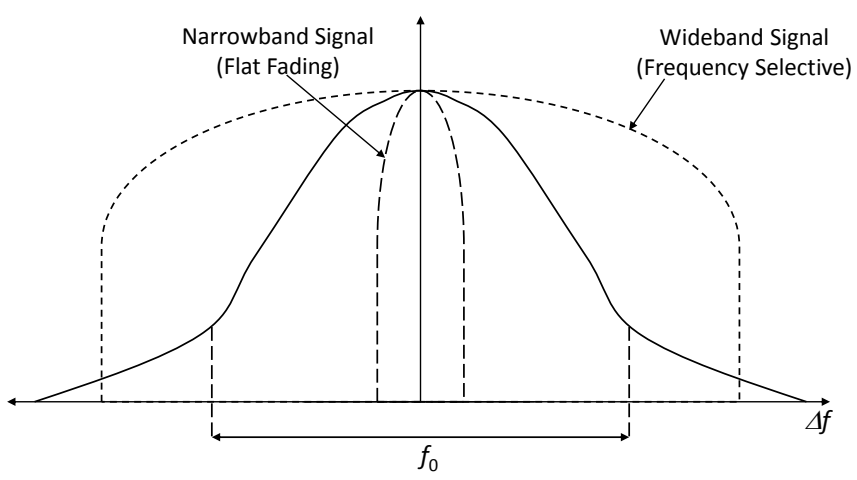

Figure 2.4: [1] Frequency-Selective Bandwidth Comparison

\section{Flat Fading Model}

The flat fading simulator is based on the fact that the fading factor of the channel is multiplicative. For this to be true, the time-varying frequency response of the communication channel $H(t, \omega)$, which for flat-fading channels is

$$
H(t, \omega)=A(t) e^{j \theta(t)}
$$

needs to be independent of frequency over the signal bandwidth, and $h(t, \tau)$ is the impulse of the channel to an impulse at time $t$, which for flat fading is

$$
h(t, \tau)=A(t) e^{j \theta(t)} \delta(\tau)
$$

where $\delta(\tau)$ is the Dirac delta function.

When this channel impulse response is used, it can be seen that the convolution performed in Equation 2.9 becomes a multiplication between the complex envelope of the modulated signal and the complex fading process. (Equation 2.11). This multiplication yields the result in Equation 2.5, which can be rewritten as

$$
s_{R}(t)=A(t) \Re\left\{s_{i}(t)\right\} \cos (\theta(t)) \cos \left(\omega_{c} t\right)-A(t) \Re\left\{s_{i}(t)\right\} \sin (\theta(t)) \sin \left(\omega_{c} t\right)
$$

Equation 2.13 can be rewritten in terms of the In-phase $(I(t))$ and Quadrature-phase $(Q(t))$

$$
s_{R}(t)=I(t) \Re\left\{s_{i}(t)\right\} \cos \left(\omega_{c} t\right)+Q(t) \Re\left\{s_{i}(t)\right\} \cos \left(\omega_{c} t+\pi / 2\right)
$$




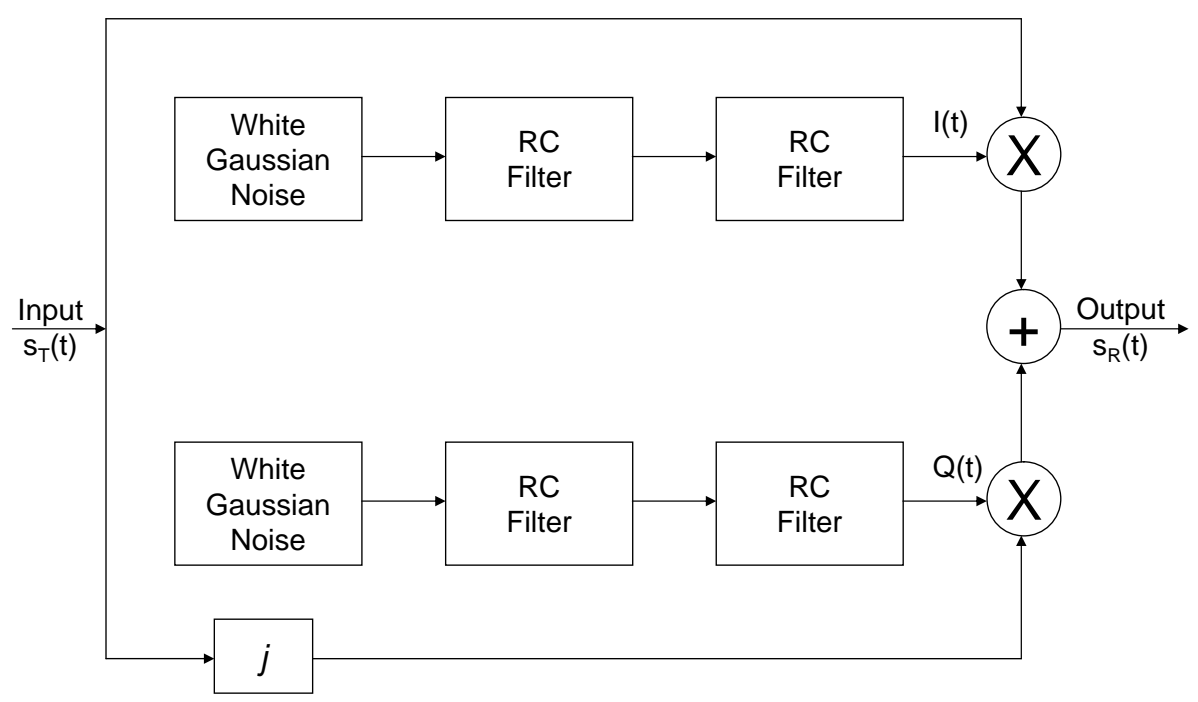

Figure 2.5: Rayleigh Flat Fading Simulator $\left(f^{-4}\right)$

where $I(t)$ and $Q(t)$ are defined in Equations 2.6 and 2.7, respectively.

These equations show that flat fading received signals can be simulated relatively simply. It can be done by generating two copies of the transmitted signal $m(t)$, shifting the phase of one of the copies by 90 degrees, and passing the signal and the phase-shifted signal through two product modulators. $I(t)$ and $Q(t)$, the modulating waveforms, represent the fading channel. Since any statistical model can be used on these waveforms, the simulator is not limited to a specific type of channel, as long as it can be shown as narrowband and nonselective over the signal bandwidth.

By using a statistical approach we can start with two zero-mean white Gaussian random processes, that are statistically independent, but have the same variance. With a given fading power spectrum, white Gaussian noise can be filtered to give desired second-order statistics. 
The processes $I(t)$ and $Q(t)$ can be correlated in some way. The correlation depends on the particular channel model. A common model is Clarke's model [11] for which the autocorrelation of $I(t)$ and $Q(t)$ will each be $R(\tau)=J_{0}\left(2 \pi f_{c} \tau\right)$. However, this model does not lend itself to simple implementation. In this thesis, we instead use a model where the power spectral density may be:

$$
S(f)=\frac{4 \tau_{R C}}{1+2\left(2 \pi \tau_{R C} f\right)^{2}+\left(2 \pi \tau_{R C} f\right)^{4}}
$$

or

$$
S(f)=\frac{16 \tau_{R C}}{3\left(1+\left(2 \pi \tau_{R C} f\right)^{2}\right)^{3}}
$$

If the PSD of a fading process is 2.15 , then it is called $f^{-4}$ fading, while if it is 2.16 it is called $f^{-6}$ fading. The $f^{-4}$ spectrum can be generated by passing white Gaussian noise through two cascaded single pole filters, while the $f^{-6}$ spectrum requires three filters. Without including a direct link from the input to the output, this statistical model will produce Rayleigh fading statistics, later this link will be discussed. Figure 2.5 shows a block diagram of the resulting $f^{-4}$ fading simulator.

In the software implementation of this simulator, a Gaussian random number generator with distribution $\mathcal{N}(0,1)$ can be used to generate both parts of the white Gaussian noise with desired variance $\sigma^{2}$ by

$$
x=\left(g_{1}+j g_{2}\right) \sigma
$$

where $g_{1}$ and $g_{2}$ are independent zero-mean samples from a white Gaussian process. The single-pole RC filters can be implemented using the recursive relationship

$$
y_{k}=a y_{k-1}+b x_{k}
$$

where $y_{k}$ is the current output of the filter, $y_{k-1}$ is the previous output, $x_{k}$ is the current input, and $\mathrm{k}$ is the sampling index. The quantities $a$ and $b$ are filter coefficients, where $a$ is determined by the filter time constant and $b$ is determined by the filter gain. This is shown in Equations 2.19 and 2.20 for $f^{-4}$ and Equations 2.19 and 2.21 for $f^{-6}$.

$$
a=e^{-\frac{T_{s}}{\tau_{R C}}}
$$




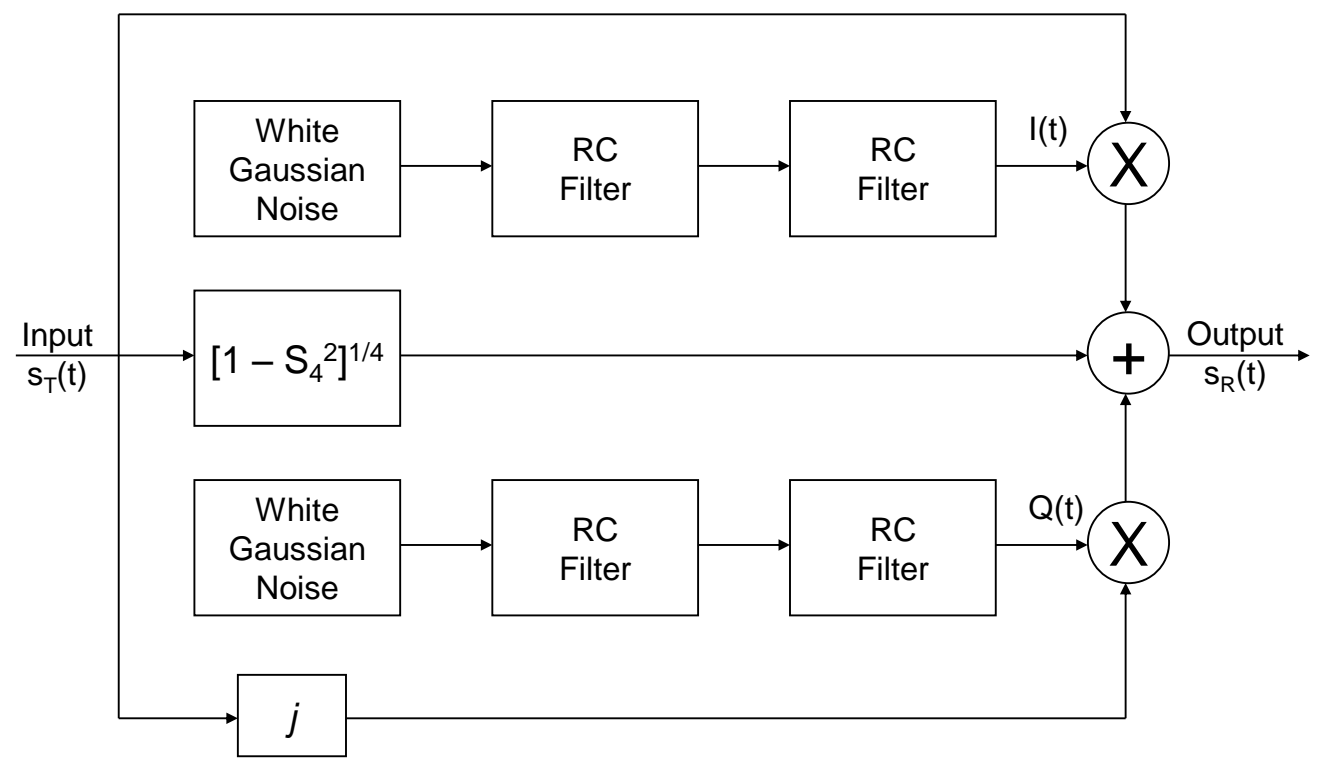

Figure 2.6: Rician Flat Fading Simulator $\left(f^{-4}\right)$

$$
\begin{gathered}
b=\left[\frac{\left(1-a^{2}\right)^{3}}{\left(1+a^{2}\right)}\right]^{\frac{1}{4}} \\
b=\left[\frac{\left(1-a^{2}\right)^{5}}{\left(1+4 a^{2}+a^{4}\right)}\right]^{\frac{1}{6}}
\end{gathered}
$$

where $\tau_{R C}$ is the RC filter time constant and $T_{s}$ is the sampling time interval. The time constant $\tau_{R C}$ is obtained by

$$
\tau_{R C}=\frac{\tau_{0}}{\beta}
$$

where $\tau_{0}$ is the decorrelation time, and $\beta$ is a constant depending on the spectrum used. For $f^{-4}, \beta=2.146193$ and for $f^{-6}, \beta=2.90463$. The normalized signal decorrelation of $\tau_{0} / T_{s}$ should generally be between 20 and 100 .

Four our implementation we combined the multiple pole filters by first taking the Z- 
transform of the difference equation of the single-pole filter:

$$
\begin{array}{r}
\mathcal{Z}\left\{y_{k}-a y_{k-1}=b x_{k}\right\} \\
Y(Z)\left(1-a z^{-1}\right)=X(Z) b \\
\frac{Y(Z)}{X(Z)}=\frac{b}{\left(1-a z^{-1}\right)}
\end{array}
$$

Since the single pole filters are cascaded, we can square the result of Equation 2.23 for $f^{-4}$ and cube it for $f^{-6}$. This will result in the following for $f^{-4}$ :

$$
H(Z)=\frac{b^{2}}{\left(1-2 a z^{-1}+a^{2} z^{-2}\right)}
$$

or for $f^{-6}$ :

$$
H(Z)=\frac{b^{3}}{\left(1-3 a z^{-1}+3 a^{2} z^{-2}-a^{3} z^{-3}\right)}
$$

where $H(Z)=Y(Z) / X(Z)$. The resulting difference equations

$$
y_{k}-2 a y_{k-1}+a^{2} y_{k-2}=b^{2} x_{k}
$$

for $f^{-4}$ fading and

$$
y_{k}-3 a y_{k-1}+3 a^{2} y_{k-2}-a^{3} y_{k-3}=b^{3} x_{k}
$$

for $f^{-6}$ fading.

As mentioned earlier, the presence of a direct path in the channel produces Rician statistics. Since part of the signal is going directly to the output Rician systems are generally less taxing than Rayleigh. This new configuration is shown in Figure 2.6.

As shown in Figure 2.6 the direct line of sight component of the system is shown as

$$
V_{s}=\sqrt{P_{0}}\left[1-S_{4}^{2}\right]^{1 / 4}
$$

where $P_{0}$ is the mean signal power of the input. The power in the random components of the system is reduced by the power in the line of sight component, so the variance of the in-phase and quadrature-phase components is calculated by

$$
\sigma^{2}=\frac{P_{0}}{2}\left[1-\sqrt{1-S_{4}^{2}}\right]
$$

In the simulator the power of the input signal is normalized so $P_{0}$ is set to unity. An example of results from the simulator can be seen in Figures 2.7 and 2.8. $S_{4}$ is the scintillation index and is always between 1 and 0 , where $S_{4}=0$ has no random components and $S_{4}=1$ has no line of sight components (Rayleigh Fading), and the values in between have Rician statistics. 


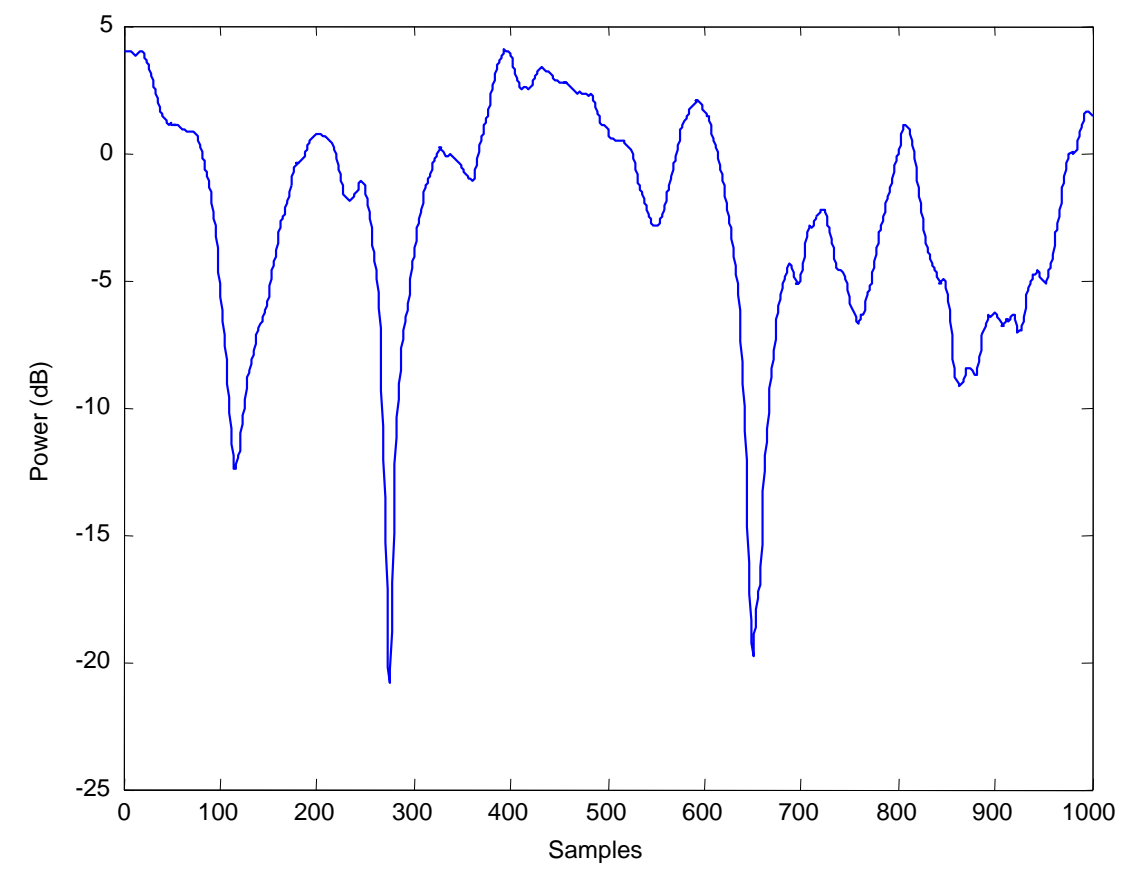

Figure 2.7: Power Amplitude of Rayleigh Fading Channel $\left(S_{4}=1, f^{-4}\right.$, and $\left.\tau_{0} / T_{s}=100\right)$

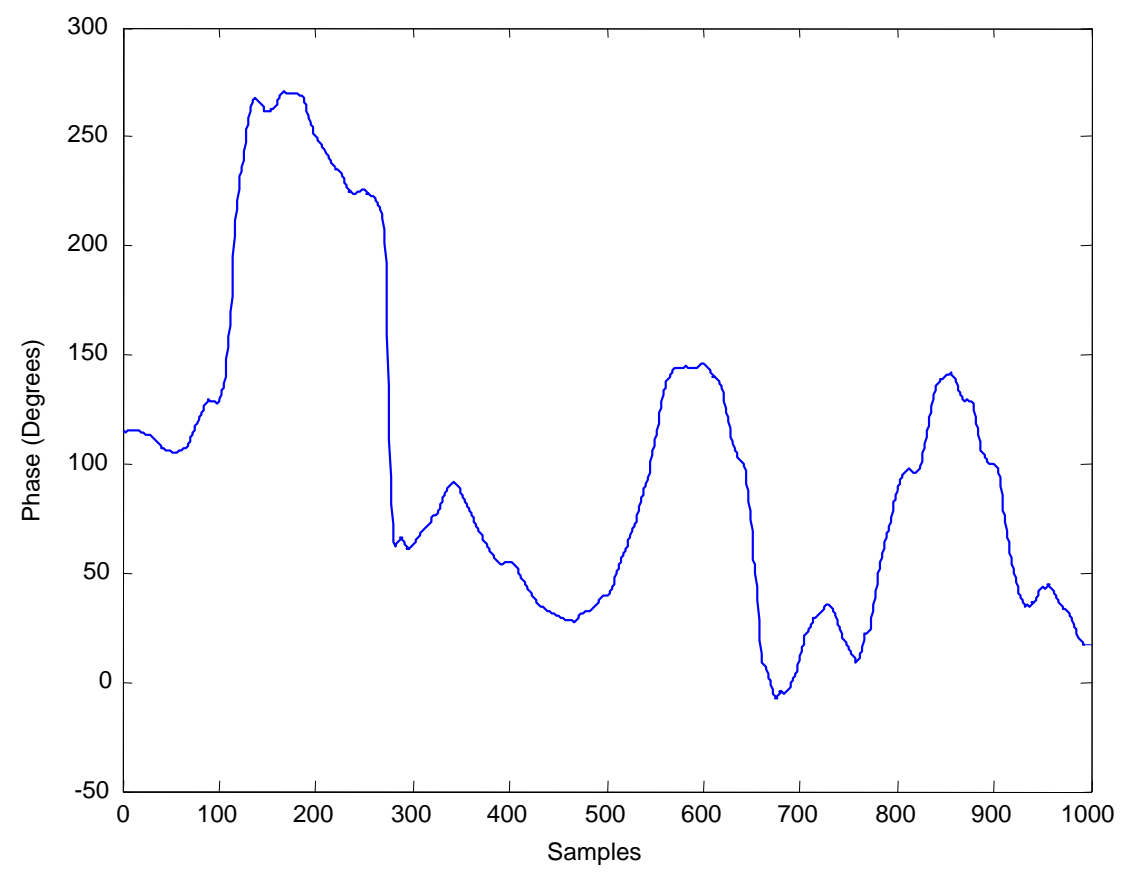

Figure 2.8: Phase of Rayleigh Fading Channel $\left(S_{4}=1, f^{-4}\right.$, and $\left.\tau_{0} / T_{s}=100\right)$ 


\section{Frequency-Selective Fading Model}

In order to generate frequency-selective fading, many flat-fading processes must be run in parallel and properly processed and combined. For the model proposed in this thesis, the power delay profile can be expressed as

$$
S_{\tau}(\tau)=2 \pi f_{0} e^{-2 \pi f_{0} \tau} u(\tau)
$$

where $f_{0}$ is the frequency-selective bandwidth of the channel, $u(\tau)$ is the unit-step function, and $\tau$ is the excess delay. Since Equation 2.30 is a continuous function of $\tau$, in order to simulate the system it is sampled at sample period $T_{s}$,

$$
S_{\tau}(\tau)=\sum_{k=0}^{K-1} P_{k} \delta\left(\tau-\tau_{k}\right)
$$

where $K$ is the required number of delay samples, $\tau_{k}=k T_{s}$ is the delay of the $k^{\text {th }}$ sample, and $P_{k}$ is the mean signal power. $P_{k}$ is obtained by integrating over one sample of Equation 2.30 giving

$$
P_{k}=e^{-2 \pi f_{0} \tau_{k}}-e^{-2 \pi f_{0} \tau_{k+1}}
$$

and $K$ is obtained by finding the minimum number of samples which contain the fraction

$P_{\tau}$ of the total received signal power. In particular $\mathrm{K}$ is found by $K=1-\left\lceil\frac{\ln \left(1-P_{\tau}\right)}{2 \pi f_{0} T_{s}}\right\rceil$, where the ceiling operator is used to guarantee that $K$ is an integer. The parameters $P_{\tau}$ and $T_{s}$ in this simulator are selectable and for results shown later, they were chosen to be $P_{\tau}=0.99$ and $T_{s}=\frac{T_{c}}{2}$, where $T_{c}$ is the chip period, which results in

$$
K=1-\left\lceil\frac{1.47}{f_{0} T_{c}}\right\rceil
$$

The impulse response of the channel which has the sampled power delay profile of Equation 2.31 is

$$
h(t ; \tau)=\sum_{k=0}^{K-1} \sqrt{P_{k}} c_{k}(t) \delta\left(\tau-\tau_{k}\right)
$$

where $c_{k}(t)$ are unit power complex Gaussian processes $\left(\mathcal{N}_{c}\right)$. In the model of Wide-Sense Stationary Uncorrelated Scattering (WSSUS) in [12], the K multi-path components in 2.34 are mutually uncorrelated processes with identical power spectrums. 
For Rician fading, the processes $c_{k}(t)$ could have nonzero-mean, but since this thesis is focusing on Rayleigh fading $\left(S_{4}=1\right)$, they have zero-mean. These processes also depend on the fading model, which in this implementation could be $f^{-4}$ or $f^{-6}$, but the focus is $f^{-4}$, which has a fading power spectrum given by Equation 2.15 where $\tau_{R C}$ is Equation 2.28 and $\beta=2.146193$.

After the HSDPA chip waveform is spread, it is shaped using a raised-cosine rolloff (RC) pulse shaping filter, which is added into our signal model. The impulse response of this RC filter is from [13] and shown by

$$
x_{R C}(\tau)=\operatorname{sinc}\left(\frac{\tau}{T_{c}}\right)\left[\frac{\cos \left(\alpha \pi \tau / T_{c}\right)}{\left(1-2 \alpha \tau / T_{c}\right)^{2}}\right]
$$

where $\alpha$ is the rolloff factor defined in the HSDPA standard [14], as $\alpha=0.22$.

The resulting channel will be a cascade of the pulse-shaping filter, with impulse response from Equation 2.35, and the actual channel, with impulse response from Equation 2.34. This equivalent channel can be represented by a convolution of these two impulse responses resulting in a new impulse response

$$
\begin{aligned}
f(t ; \tau) & =x_{R C}(\tau) * h(t ; \tau) \\
& =x_{R C}(\tau) * \sum_{k=0}^{K-1} \sqrt{P_{k}} c_{k}(t) \delta\left(\tau-\tau_{k}\right) \\
& =\sum_{k=0}^{K-1} \sqrt{P_{k}} c_{k}(t) x_{R C}\left(\tau-\tau_{k}\right)
\end{aligned}
$$

where the last step assumes that $c_{k}(t)$ changes slowly with respect to the chip period $T_{c}$.

As is indicated in the last term of Equation 2.36, there is some ISI. If left alone this ISI will cause severe quality loss in the signal,but the frequency-selectivity of the channel can be used as a type of diversity, if the processing is done properly. The received signal can be processed through an equalizer, or a rake receiver. The equalizer implementation is typical of commercial HSDPA setups, which is due to the Walsh codes that are used having poor autocorrelation properties not cooperative with rake receiving.

Proposal of not using the typical $5 \mathrm{GHz}$ signal bandwidth limitation of commercial HSDPA, would allow for a secondary spread of the signal. The additional spreading will allow 
for the elimination of an equalizer and to be able to process using a rake receiver. A length 11 Barker code is proposed as the secondary spreader, represented by the sequence

$$
\mathbf{x}_{b c}=[+1,+1,+1,-1,-1,-1,+1,-1,-1,+1,-1]
$$

Use of the barker code increases the chip rate and signal bandwidth by a factor for 11, so that the bandwidth will now be $11(1+\alpha) R_{c}=51.5 \mathrm{MHz}$, where $R_{c}=3.84 \mathrm{Mchips} /$ second as indicated by the HSDPA standard. Also, Barker codes are known to have good autocorrelation properties.

By using this code, with good spreading properties, signal components separated by at least one chip period $T_{c}$ can be resolved. The use of an $L$-fingered chip matched rake receiver, with $L$ between 1 and 4 , is used to reconcile the signal. The gain of the channel $g_{l}(t)$ of the $l^{\text {th }}$ signal finger, $0 \leq l \leq L-1$, is found by sending the signal through a chip-matched filter and sampling the output with delay $l T_{c}$. The complex channel gain now seen by the $l^{\text {th }}$ finger is

$$
\begin{aligned}
g_{l}(t) & =f(t ; \tau) \\
& =\sum_{k=0}^{K-1} \sqrt{P_{k}} c_{k}(t) x_{R C}\left(l T_{c}-\tau_{k}\right)
\end{aligned}
$$

The fingers of the rake receiver are interdependent, even though the $K$ fading processes $c_{k}(t)$ are independent. This is due to the bandlimiting effect of the pulse shaping filter.

Maximal Ratio Combining (MRC) is used on the output of the $L$ fingers, and gives a real valued channel amplitude gain

$$
a_{M R C}(t)=\sqrt{\sum_{l=0}^{L-1}\left|g_{l}(t)\right|^{2}}
$$

This gain is sampled at the symbol rate $R_{s}$, where the channel amplitude gain of the $i^{\text {th }}$ signal becomes

$$
\begin{aligned}
a_{M R C}[i] & =a_{M R C}\left(i T_{s}\right) \\
& =\sqrt{\sum_{l=0}^{L-1}\left|g_{l}\left(i T_{s}\right)\right|^{2}} \\
& =\sqrt{\sum_{l=0}^{L-1}\left|\sum_{k=0}^{K-1} \sqrt{P_{k}} c_{k}[i] x_{R C}\left(l T_{c}-\tau_{k}\right)\right|^{2}}
\end{aligned}
$$


where $c_{k}[i]$ is the $i^{t h}$ sample of the process $c_{k}(t)$ when sampled at the symbol rate.

Equation 2.40 describes how the channel is simulated. A total of $K$ independent fading simulators are run in parallel, producing the sequences $c_{k}[i]$. Each fading sequence is a zeromean complex Gaussian process with power spectrum given by Equation 2.15 and sampled at the symbol rate. For each finger of the rake receiver, the $K$ channel gain samples are combined by sampling Equation 2.38 at the symbol rate. The $L$ fingers are then MRC combined according to Equation 2.39.

In our problem, we can use multiple antennas on the ground. Where $Q$ is the number of ground antennas used. Assuming the $Q$ antennas are widely spaced so as to have the fading seen by each be statistically independent, the signals can be combined in many ways. If the antennas are connected to $L$-branch rake receivers, the system could be shown as a $Q L$-branch rake receiver. However, this method requires information to be shared among the $Q$ antennas, and would be difficult if the antennas are space far apart. If the system combines the signals from the antennas after demodulation, it would require less information to be shared.

Using this method, the output of each antenna's rake receiver will first be passed through a demodulator whose implementation will be shown in Section 4.1 Equation 4.1. Let $\lambda_{j}^{(q)}$ be the $\log$ likelihood ratio (LLR) of the $j^{\text {th }}$ code bit $c_{j}$ at the output of the $q^{\text {th }}$ demodulator of the antenna. The LLRs from the $Q$ antennas are then combined by simple addition forming the overall LLR

$$
\lambda_{j}=\sum_{q=0}^{Q-1} \lambda_{j}^{(q)}
$$

This combined LLR is then passed through the decoder as usual. If an outage analysis is being performed, mutual information is measured between the input codeword and LLR as defined in Equation 2.41. This implementation is beneficial because most of the processing can be placed close to the antenna, such as channel estimation, synchronization, rake reception, and demodulation. Central processing would only need to be performed for turbo decoding and decompression of the images. 


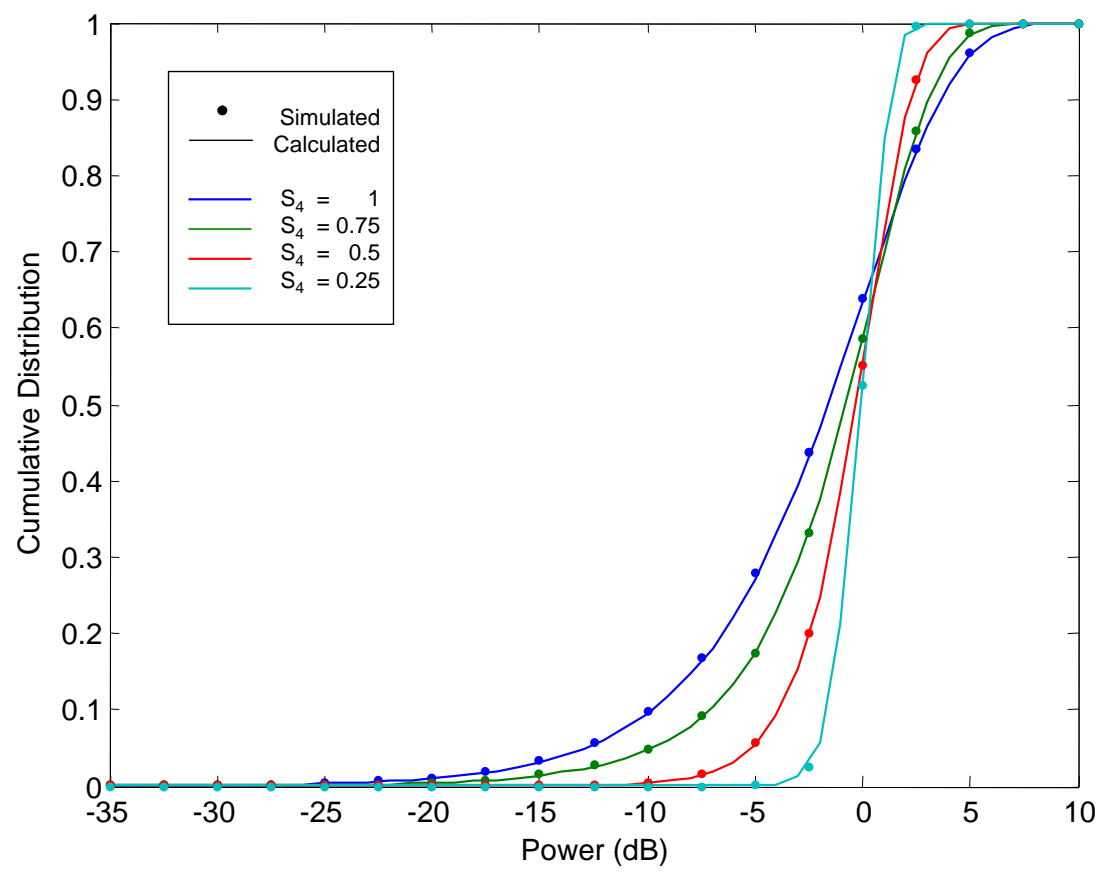

Figure 2.9: Cumulative Distribution Function of Rician Fading, $f^{-4}$ Doppler Spectrum, and $\tau_{0} / T_{s}=100$

\subsubsection{Validation}

The validation of our fading model is performed using statistical models from [9].

\section{Cumulative Distribution Function}

The cumulative distribution function $(\mathrm{CDF})$ is defined as the probability that the random process will have a value less than the functions argument. The CDF of $\mathrm{P}$, where $\mathrm{P}$ is the instantaneous power of the fading channel, is identified as

$$
F(P)=e^{-\frac{R}{1-R}} \sum_{n=0}^{\infty} \frac{1}{\Gamma^{2}(n+1)}\left(\frac{R}{1-R}\right)^{n} \gamma\left(n+1, \frac{P / P_{0}}{1-R}\right)
$$

where, $R$ is the Rician Index defined as $\sqrt{1-S_{4}^{2}}, P$ is the Power of the fading channel, $\Gamma(\cdot)$ is the gamma function, and $\gamma(\cdot)$ is the incomplete gamma function. This is a form of the 


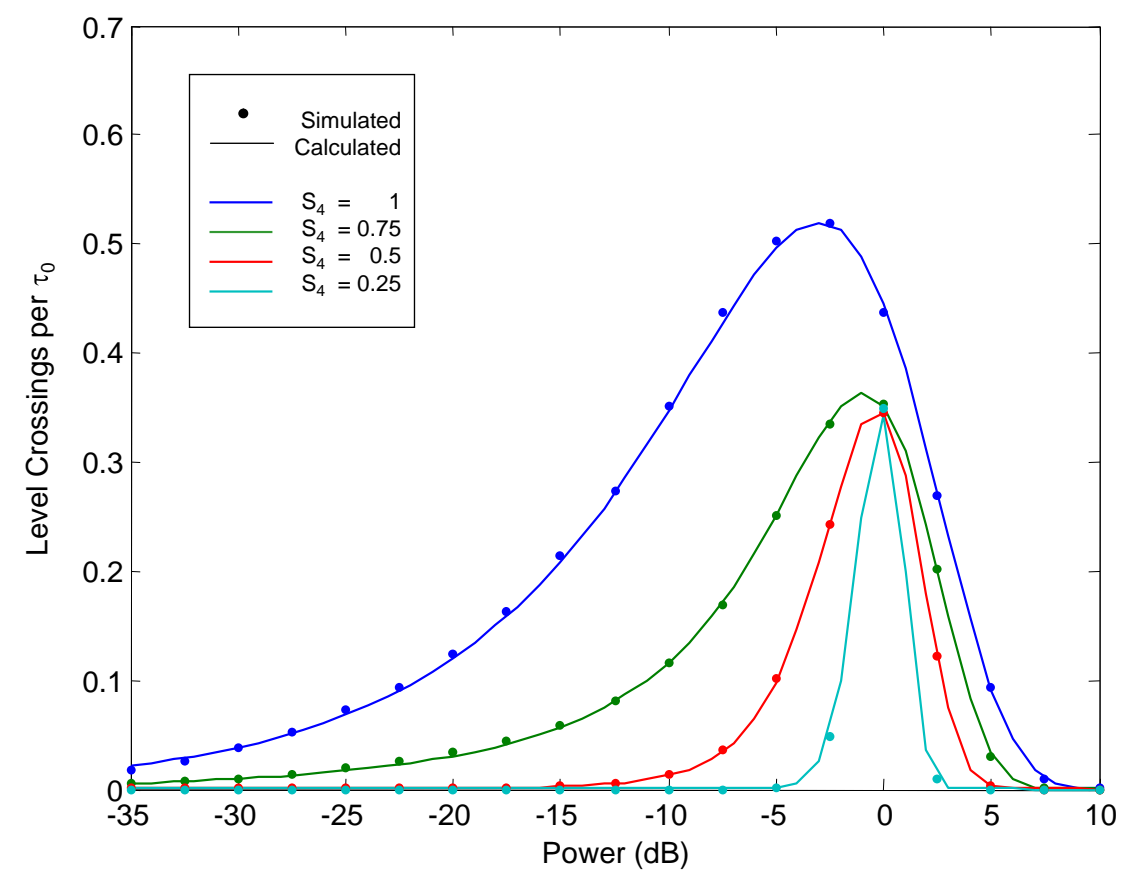

Figure 2.10: Mean Up Level Crossing Rate of Rician Fading, $f^{-4}$ Doppler Spectrum, and $\tau_{0} / T_{s}=100$

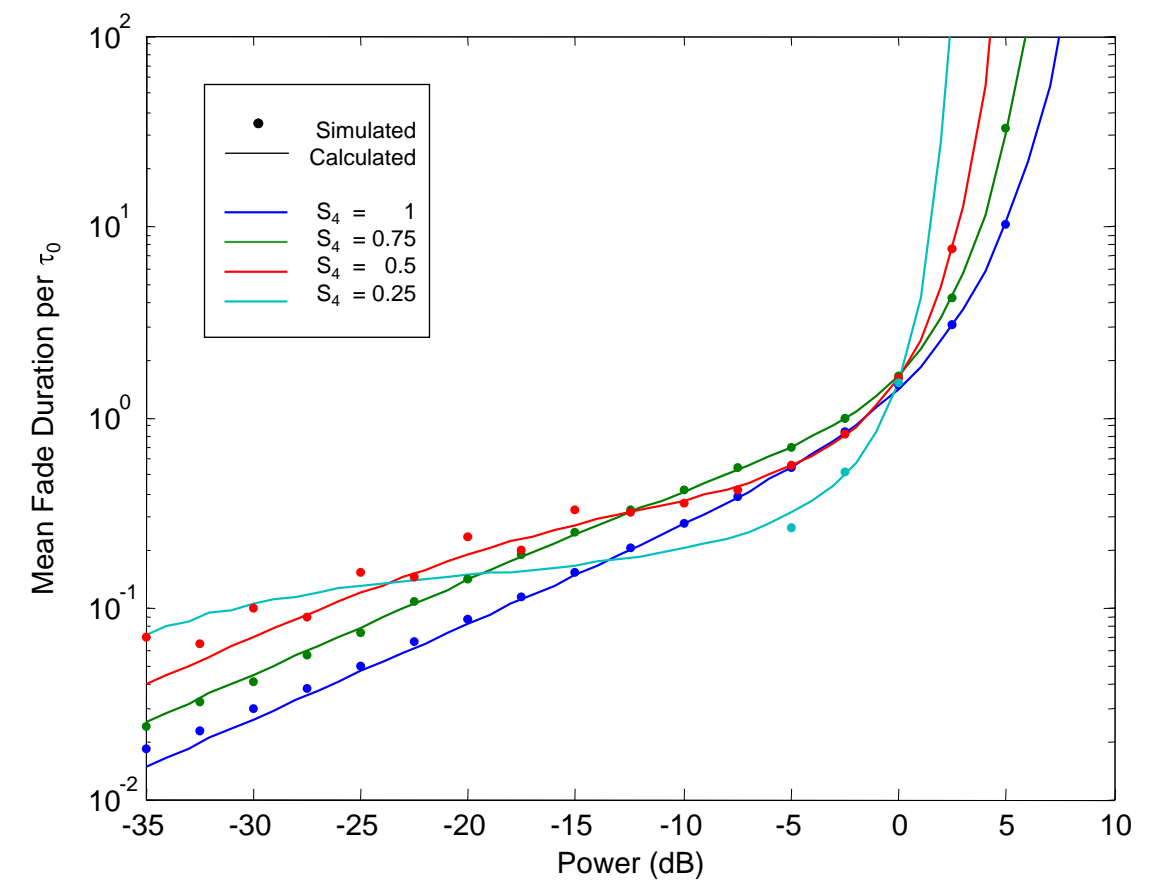

Figure 2.11: Mean Fade Duration of Rician Fading, $f^{-4}$ Doppler Spectrum, and $\tau_{0} / T_{s}=100$ 
Marcum Q-Function, and can be evaluated by [10]

$$
F(P)=1-Q_{1}\left(\sqrt{\frac{R}{\sigma^{2}}}, \sqrt{\frac{P}{\sigma^{2}}}\right)
$$

and in Rayleigh fading this can be reduced to

$$
F(P)=1-e^{-P}
$$

where both of these equations are evaluated when the mean power $P_{0}$ is normalized to 1 .

To determine the CDF of our simulated channel the total number of samples below a give power level is divided by the total number of samples generated shown

$$
F\left(P_{i}\right)=n_{i} / N
$$

where $P_{i}$ is the power level threshold, $n_{i}$ is the number of samples below that threshold, and $N$ is the total number of samples in the simulated channel. This equation will approximate the calculated value from Equation 2.42 as long as $\mathrm{N}$ is sufficiently large. The comparison between our simulator and the calculated value is shown in Figure 2.9.

\section{Mean Level Crossing Rate}

The mean level crossing rate is the average number of times per some interval $T$ the fading channel crosses a given power level. The calculated value for Rician fading statistics is given as

$$
\langle N(P, T)\rangle=\Delta\left(\frac{T}{\tau_{0}}\right) \sqrt{\frac{8 P}{\pi(1-R)}} e^{-\frac{P+R}{1-R}} I_{0}\left(\frac{2 \sqrt{R P}}{1-R}\right)
$$

where $\Delta$ is a constant depending on the frequency spectrum used. $\Delta=1.5176$ for $f^{-4}$ and $\Delta=1.1858$ for $f^{-6}$. In order to get the mean crossing rate in only one direction Equation 2.46 will be divided by 2 .

When determining the mean level crossing rate per $\tau_{0}$ by calculating the number of times the fading channel crosses a certain power threshold $P_{i}$. This number is then divided by the total number of samples $N$, and multiplied by the ratio $\tau_{0} / T_{s}$ (normalized signal decorrelation), which is 100 for the simulations shown as

$$
\left\langle N\left(P_{i}, \tau_{0}\right)\right\rangle=\frac{n_{i} \tau_{0} / T_{s}}{N}
$$


where Equation 2.47 can be divided by 2 in order to get level crossing only in one direction. The comparison of the simulator and the calculated value is shown in Figure 2.10.

\section{Mean Fade Duration}

The mean fade duration, is the amount of time over the interval the power $P$ of the simulator is below a given power threshold. This is can be computed by

$$
\left\langle T_{\text {Dur }}(P)\right\rangle=\frac{2 \tau_{0} F(P)}{\langle N(P, \tau)\rangle}
$$

Equation 2.48 can also be used to determine the simulated mean fade duration also, by changing the function to be in terms of $P_{i}$. A comparison is shown in Figure 2.11 


\section{Chapter 3}

\section{Source to Relay Link}

\subsection{HSDPA Overview}

Both link designs in this system (Source to relay, and relay to destination) are based on High-Speed Downlink Packet Access (HSDPA) Technology. HSDPA is a standard for high-speed downlink access currently used in third generation $(3 \mathrm{G})$ cellular phone systems, and is currently deployed by AT\&T with peak downlink data rate of 3.6 Mbps. T-Mobile is also in the process of deploying this type of system. HSDPA is part of the Universal Mobile Telecommunications System (UMTS) group of standards, developed by the European Telecommunications Standards Institute (ETSI) and promoted by the Third Generation Partnership Project (3GPP). The standards documents used to develop this thesis are 25.212 [15], which shows the baseband processing and turbo coding, 25.213 [16], which shows the spreading and modulation, and 25.104 [14], which shows the radio requirements, including the chip shaping filter already shown in Section 2.3.1 Equation 2.35. Document 25.101 [17] is also used, and gives standardized reference link designs that will be used on the second link of the system (relay to destination).

A high level diagram of the baseband processing done by an HSDPA transmitter is shown in Figure 3.1. In the HSDPA standard, time is separated into frames called transmission time intervals (TTIs), which are each 2 msec long. Once data message is turbo encoded, modulated, and spread during each TTI.

Before turbo encoding, a 24-bit cyclic redundancy check (CRC) is added to the end of 


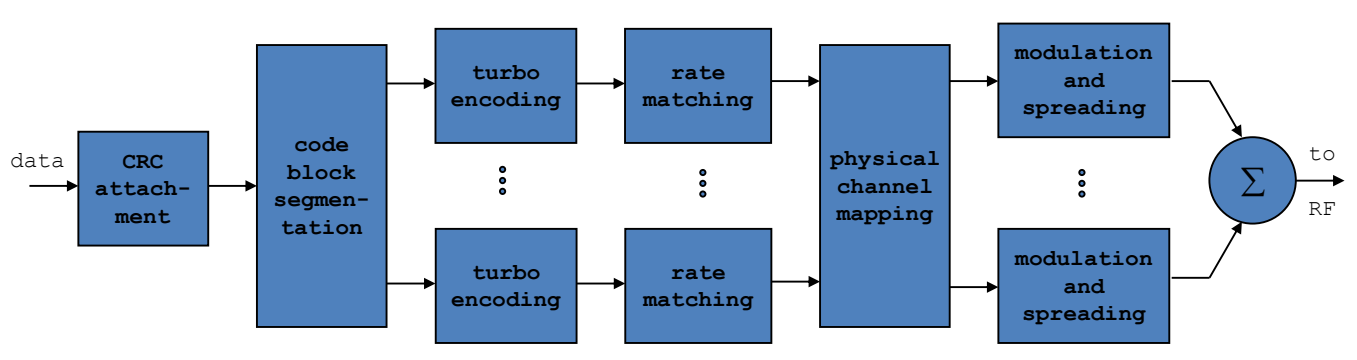

Figure 3.1: Baseband processing at HSDPA transmitter

the data. This CRC is used by the receiver to detect uncorrected errors. The turbo encoder, has an internal bit interleaver that can be set to any length between 40 and 5114, including the bounds. If the CRC-encoded message is longer than 5114 bits, it must be broken up into equal length code blocks. If the number of bits in the CRC-encoded message are not divisible by the number of code blocks required, some filler bits will be added to the message before they are broken apart. Also, a maximum of 5 code blocks can be sent over each TTI.

After the equal length code blocks are obtained, each one is passed to the turbo encoder represented in Figure 3.2. The encoder is made up of two recursive systematic convolutional (RSC) built-in encoders, and a turbo interleaver (described previously). The RSC encoders produce a sting of parity bits $\left(Z_{i}\right.$ and $Z_{i}^{\prime}$ from Figure 3.2), that are combined with the systematic data stream ( $X_{i}$ in Figure 3.2), and triple the length of the code block. After the block is encoded 12 tail bits are attached to the end, where these bits are obtained by throwing the switch in Figure 3.2, and running the system for three extra cycles. The code rate, as a result of this process, is

$$
R=\frac{K}{3 K+12}
$$

where $\mathrm{K}$ is the length of the segmented code block.

If a code rate different from the result in Equation 3.1 is required, it can be adjusted through rate matching. This process creates higher rates by puncturing code bits and lower rates by repeating code bits. The turbo encoded blocks are passed through a rate-matching 


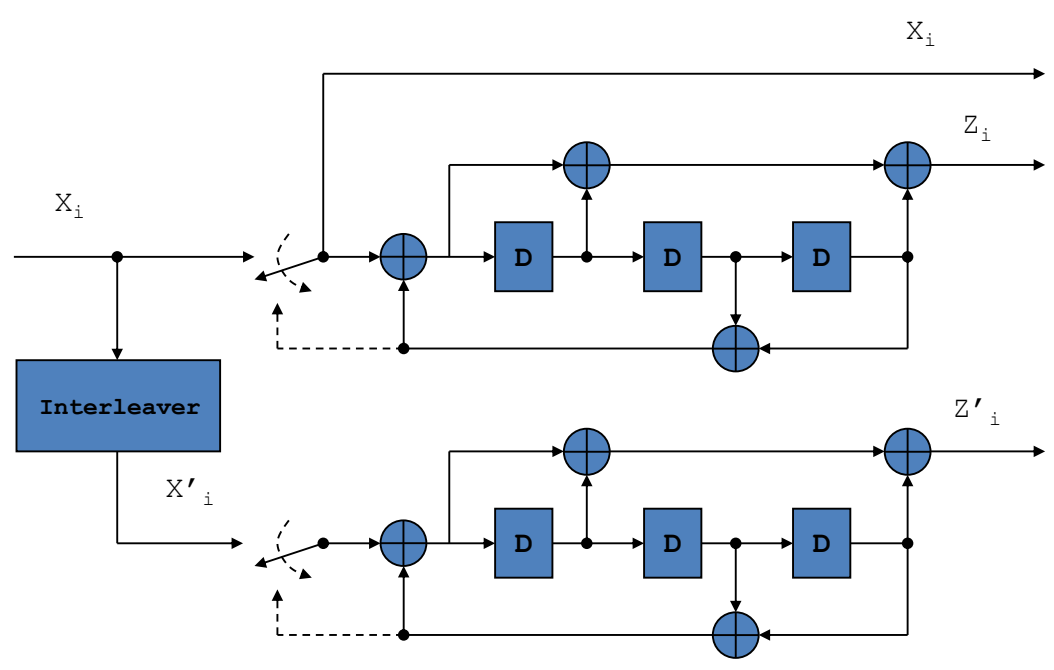

Figure 3.2: UMTS Turbo Encoder

processer, as shown in Figure 3.1, which will give the desired code rate. These rate-matched code blocks are modulated and spread. The HSDPA standard uses direct sequence spreadspectrum (DSSS) with a chip rate of $R_{c}=3.84 \mathrm{Mchips} / \mathrm{sec}$, and supports both QPSK and 16-QAM (Quadrature Amplitude Modulation).

The signal is spread using one or more Walsh codes of length 16, of which there are 16. Also, the set of Walsh codes is mutually orthogonal, which effectively separates the channel into 16 orthogonal subchannels. These subchannels are called physical channels in the HSDPA standard. One channel is always reserved as a pilot channel, to help with coherent reception and channel estimation. Any or all of the remaining 15 channels may be used to transmit the rate-matched code blocks, as long as there are an integer number of physical channels per code block. In Figure 3.1, the rate-matched code blocks are mapped to the $P$ physical channels, where $1 \leq P \leq 15$. In previous generation DSSS systems only one code was allowed per user.

Each physical channel can be modulated using either QPSK or 16-QAM, but all $P$ 
channels must use the same modulation. The symbol rate $\left(R_{s}\right)$ for each physical channel is

$$
R_{s}=\frac{R_{c}}{16}
$$

where for our purposes $R_{c}=3.84 \mathrm{Mchips} / \mathrm{sec}$, making $R_{s}=240 \mathrm{kbaud}$. This will make the overall symbol rate for the link $240 P$ kbaud. Since each TTI is 2 msec long, there are 480 symbols per physical channel per TTI. When QPSK (2 bits per symbol) modulation is used, $2(480)=960$ code bits per physical channel per TTI can be transferred. Also, when 16-QAM (4 bits per symbol) modulation is used, 4(480) = 1920 code bits per physical channel per TTI can be transferred. The maximum theoretical rate that code bits can be transmitted when using 16-QAM and all 15 available physical channels is 15(1920)/(2 msec) $=14.4 \mathrm{Mbps}$. The actual data rate will be lower, due to rate matching and the number of physical channels used, but can be calculated by dividing the number of data bits encoded per TTI by 2 msec.

As shown in Figure 3.1, the spread physical channels are summed and then passed to the RF modulator. Before RF modulation, the spread and code modulated symbols are passed through a chip shaping filter. This chip-shaping is done through the use of RC pulse shaping as described in Equation 2.35, where $\alpha=0.22$. This results in RF bandwidth of $R_{c}(1+\alpha) \approx 4.7 \mathrm{MHz}$. Multiple HSDPA links can be supported in a typical cell network, and would be separated using $5 \mathrm{MHz}$ channel spacings.

HSDPA also has the option of using Hybrid Auto Repeat-Request (HARQ). This allows a retransmission request by sending a negative acknowledgement (NACK) back to the transmitter, if a receiver is not able to correct a received TTI. The correction of the code performed by the receiver can be checked using the CRC error detection code that was attached before it was transmitted.

If a NACK is sent and retransmission is to be performed, there are two separate modes that the system can operate in. One mode has the receiver send the exact same encoded TTI, and the receiver will combine the retransmitted packet with the original by adding the code bit log-likelihood ratios before passing to the turbo decoder. This mode is called Chase Combining (CC). Also, the transmitter could encode the message differently and a different set of parity bits can be sent. This can be done by having the rate-matching processor 


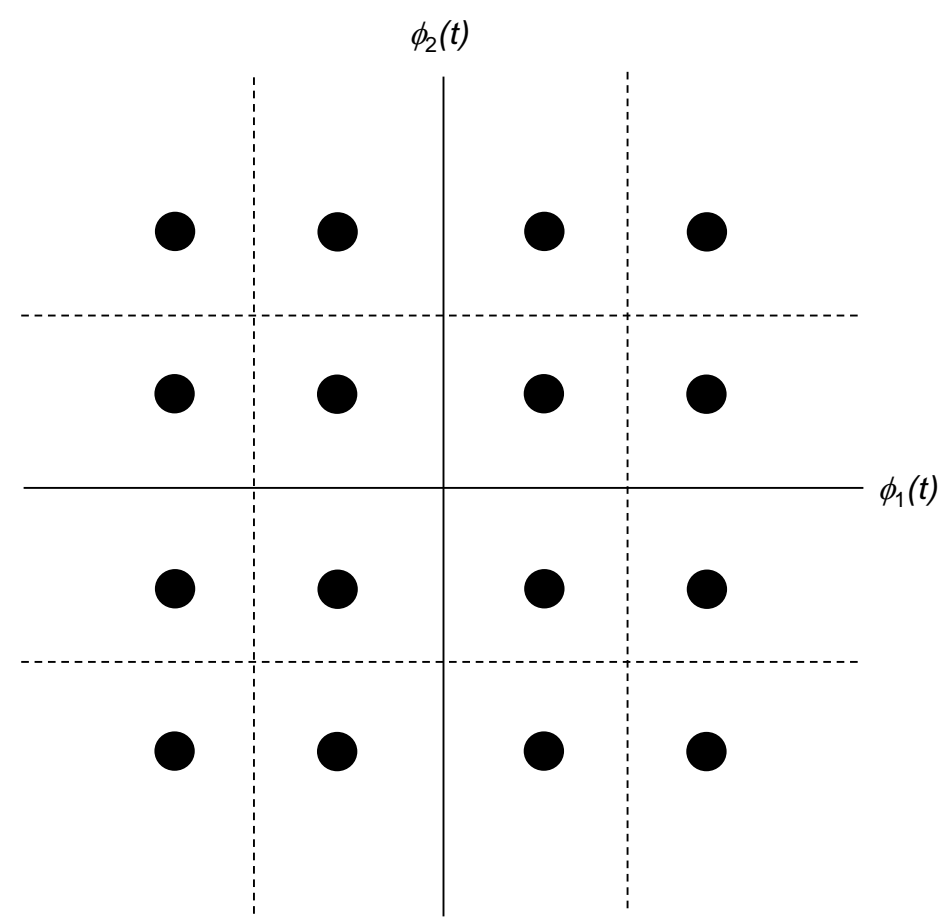

Figure 3.3: 16-QAM Signal Constellation

puncture a different set of bits than was punctured in the original. This mode is called incremental redundancy (IR).

If this first retransmission fails, another could be requested, and if that also fails, then a third would be requested, giving a total of four transmissions. While more requests could theoretically be made, the standard 25.101 [17] limits the number of HARQ attempts to four.

The way in which constellation symbols are labeled with code bits is called a symbollabeling map. This labeling has no performance impact on QPSK, due to every signal having 2 neighbors as shown in Figure 2.2, but 16-QAM is highly susceptible to this mapping. In the mapping for QAM, points on the interior each have four neighbors, making them more vulnerable to noise, than border points, who have two neighbors if they are a corner point and three otherwise. This can be seen in Figure 3.3, and means that the error rate of the signals will not be equal. When the system is run in IR mode, and using 16-QAM modulation, 


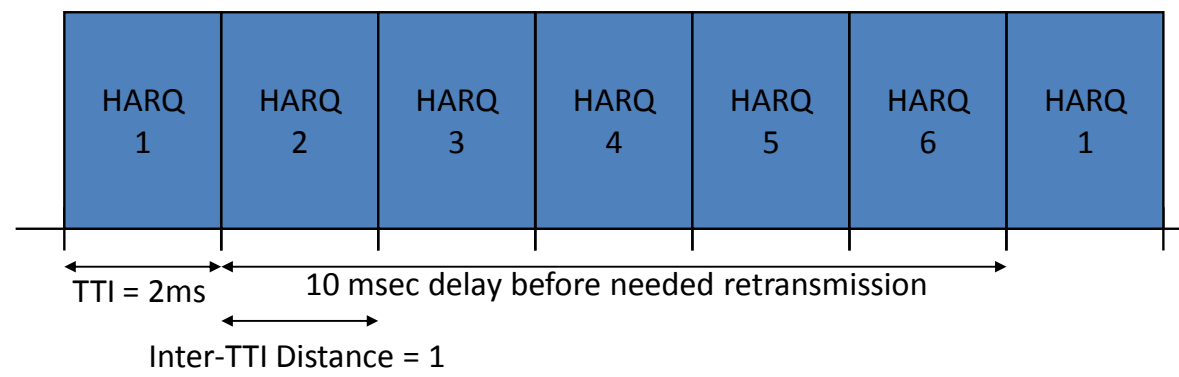

Figure 3.4: H-Set 6 HARQ Interleaving with 6 processes

diversity can be added through mapping rearrangement, which will use a different 16-QAM labeling map for each IR retransmission. This strategy is based on that fact that if the mapping used in a previous transmission was "bad" for the codeword sent, a rearranged mapping may be better to use for the retransmission.

Due to delays from propagation and processing, there will be a non-negligible delay from the time from transmission of the TTI to the reception of the ACK/NACK. To more efficiently use the channel, HSDPA allows for the interleaving of multiple HARQ processes. For instance, the H-Set 6 reference design of 25.101 [17], allows 6 HARQ processes to run concurrently with an inter-TTI distance of 1 as shown in Figure 3.4. In this design, the transmitter can service 5 other ARQ processes while waiting for the ACK/NACK of a particular TTI. This allows for a $10 \mathrm{msec}$ delay between the end of the transmission for a TTI until it must be retransmitted, where this delay must account for the round-trip propagation delay, receiver processing time, and the transmitters processing of the ACK/NACK.

The use of HARQ is not proposed for the link between source and relay, because the close distance does not allow enough time for a retransmission. However, because of these shorter distances one turbo coded transmission should be sufficient. For the longer link from relay to destination, HARQ will be used because more time will be available allowing for additional time diversity to help compensate for possible severe fading in the link. 


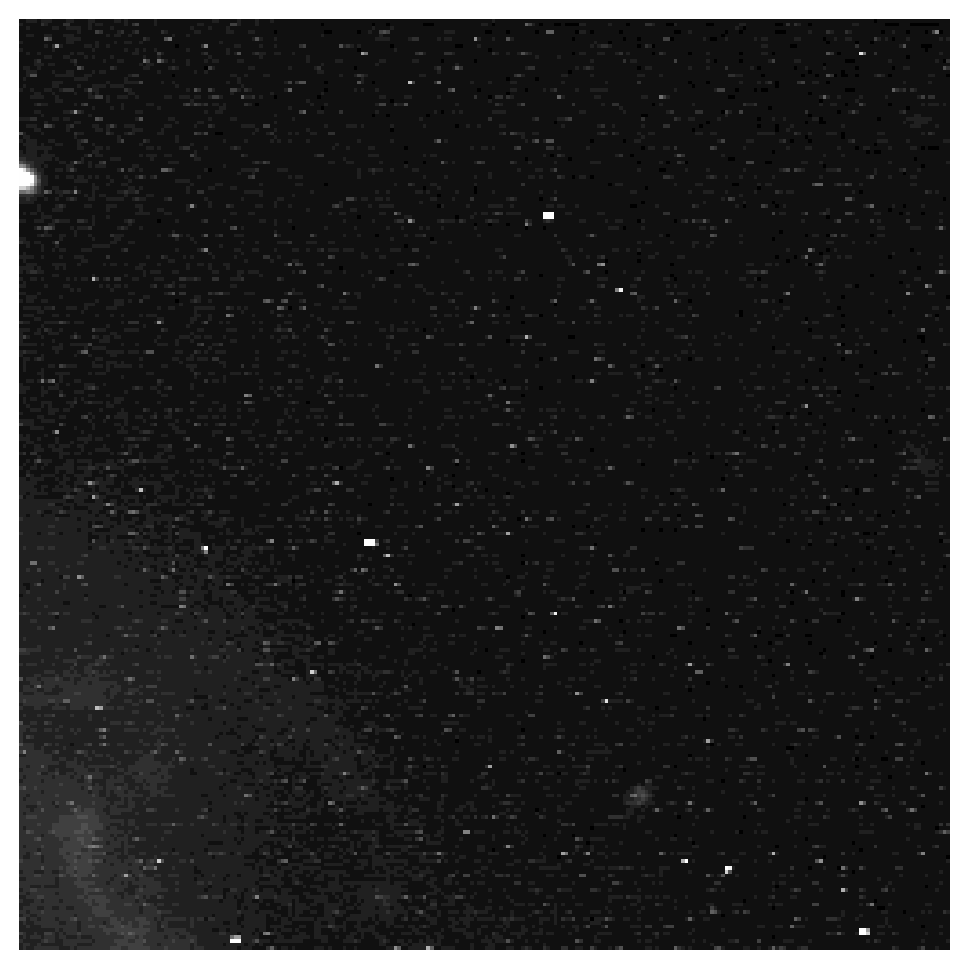

Figure 3.5: 256 by 256 grey-scale image using 4 bit quantization

\subsection{Source to Relay Link}

The source of the system contains a sensor for taking grey-scale images with a pixel resolution of 256 by 256 using 4 bits of quantization. An example of an image taken in this way is shown in Figure 3.5. The size of images taken using this method are 262,144 bits, and will also be stamped with the time taken, along with the location, orientation, and identity of the source. Instead of sending the full location information with each image, only the differences from the last transmission will be sent. This information was assumed that it would only increase the size of the image packet to 270 kbits.

The link design from source to relay is based on the HSDPA standard, as discussed in Section 3.1. The theoretical limit of the HSDPA data rate is $14.4 \mathrm{Mbps}$, but that is only achievable by turning off turbo code (performed by puncturing all parity bits during ratematching), using no HARQ, the 16-QAM modulation, and using the maximum number of 


\begin{tabular}{|l|c|}
\hline \# Physical Channels & $P=14$ \\
\hline Required Data Rate & $2.7 \mathrm{Mbps}$ \\
\hline Message Bits per TTI (Data Rate)(2 msec) & $5400 \mathrm{bits}$ \\
\hline Post CRC Message Length & 5424 bits \\
\hline Required \# Code Blocks & 2 \\
\hline Code Block Length & 2712 bits \\
\hline $\begin{array}{l}\text { Post Encoding and Rate Matching } \\
\text { Turbo Codeword Length }\end{array}$ & 6720 bits \\
\hline \# Physical Channels per Codeword & 7 \\
\hline Code Rate $\frac{\text { Message Bits perTTI }}{\text { (\# Code Blocks)(Turbo Codeword Length) }}$ & 0.4018 \\
\hline
\end{tabular}

Table 3.1: QPSK at 10 fps Video Transmission Rate

\begin{tabular}{|l|c|}
\hline \# Physical Channels & $P=15$ \\
\hline Required Data Rate & $5.4 \mathrm{Mbps}$ \\
\hline Message Bits per TTI (Data Rate) $(2 \mathrm{msec})$ & $10800 \mathrm{bits}$ \\
\hline Post CRC Message Length & $10824 \mathrm{bits}$ \\
\hline Required \# Code Blocks & 3 \\
\hline Code Block Length & $3608 \mathrm{bits}$ \\
\hline $\begin{array}{l}\text { Post Encoding and Rate Matching } \\
\text { Turbo Codeword Length }\end{array}$ & $4800 \mathrm{bits}$ \\
\hline \# Physical Channels per Codeword & 5 \\
\hline Code Rate $\frac{\text { Message Bits per TTI }}{(\text { \# Code Blocks })(\text { Turbo Codeword Length })}$ & 0.75 \\
\hline
\end{tabular}

Table 3.2: QPSK at 20 fps Video Transmission Rate

Walsh codes (15 due to only having a maximum of 15 available physical channels). This type of link would provide no protection from errors. By using the turbo code and transmitting at a lower data rate error performance can be improved greatly.

Four link configurations were considered, that can achieve video rates of between 10 and 28 frames per second (fps). None of these links use HARQ, so the video frame rate will be kept constant. These links are described in Tables 3.1, 3.2, 3.3, and 3.4.

Higher video rates could be supported, but not as efficiently. For examples, in order to obtain a frame rate of $29 \mathrm{fps}$, the message would have to be split into four code blocks. Each code block would be mapped to 3 physical channels causing only 12 of the 15 available to be used. In the end, the turbo code would operate at the code rate of 0.68 . This would be an increase in code rate by approximately $30 \%$, while the increase in frames per second would 


\begin{tabular}{|l|c|}
\hline \# Physical Channels & $P=15$ \\
\hline Required Data Rate & $5.4 \mathrm{Mbps}$ \\
\hline Message Bits per TTI (Data Rate) $(2$ msec $)$ & $10800 \mathrm{bits}$ \\
\hline Post CRC Message Length & 10824 bits \\
\hline Required \# Code Blocks & 3 \\
\hline Code Block Length & 3608 bits \\
\hline $\begin{array}{l}\text { Post Encoding and Rate Matching } \\
\text { Turbo Codeword Length }\end{array}$ & 9600 bits \\
\hline \# Physical Channels per Codeword & 5 \\
\hline Code Rate $\frac{\text { Message Bits per TTI }}{\text { (\# Code Blocks)(Turbo Codeword Length) }}$ & 0.375 \\
\hline
\end{tabular}

Table 3.3: 16-QAM at $20 \mathrm{fps}$ Video Transmission Rate

\begin{tabular}{|l|c|}
\hline \# Physical Channels & $P=15$ \\
\hline Required Data Rate & $7.56 \mathrm{Mbps}$ \\
\hline Message Bits per TTI (Data Rate) $(2$ msec) & $15120 \mathrm{bits}$ \\
\hline Post CRC Message Length & $15144 \mathrm{bits}$ \\
\hline Required \# Code Blocks & 3 \\
\hline Code Block Length & $5048 \mathrm{bits}$ \\
\hline $\begin{array}{l}\text { Post Encoding and Rate Matching } \\
\text { Turbo Codeword Length }\end{array}$ & $9600 \mathrm{bits}$ \\
\hline \# Physical Channels per Codeword & 5 \\
\hline Code Rate $\frac{\text { Message Bits perTTI }}{\text { (\# Code Blocks)(Turbo Codeword Length) }}$ & 0.5250 \\
\hline
\end{tabular}

Table 3.4: 16-QAM at 28 fps Video Transmission Rate 
only be about $3 \%$.

The four links described in Tables 3.1 to 3.4 were simulated over an AWGN channel. The turbo decoder ran 14 iterations of either the log-MAP or max-log-MAP decoding algorithm [18]. Generally, the performance of log-MAP decoding will be better than max-log-MAP, but is more complex to implement. The CRC code is used to half the decoder once the codeword is corrected, removing over-iteration.

Figure 3.6 shows the performance of two QPSK-based links. The figure shows the bit error rate $(\mathrm{BER})$ as a function of $E_{s} / N_{0}$. For the $10 \mathrm{fps}$ system, using log-MAP decoding provides a $0.4 \mathrm{~dB}$ improvement over max-log-MAP, while the increase in the 20 fps system is only approximately $0.2 \mathrm{~dB}$. In order to double the framerate from 10 to $20 \mathrm{fps}$ requires a more than doubling of the power (Increase of $4.6 \mathrm{~dB}$ ). A slight error floor develops for the 20 fps system at BER $10^{-5}$, which can be attributed to the rate-matching processer needing to puncture a large number of parity bits, causing the code to weaken.

Figure 3.7 shows the performance of two 16-QAM based links, that can achieve rates of $20 \mathrm{fps}$ and $28 \mathrm{fps}$. In comparison to the $20 \mathrm{fps}$ QPSK-based link (also pictured in Figure 3.7), the 20 fps QAM-based system does have better performance. The 20 fps QAM-based system also shows a flattening error floor, but remains steaper than the floor of the QPSKbase system. This is attributed to the QAM-based system operating at a lower code rate than the QPSK-based system, which requires fewer parity bits to be punctured by the ratematching processor. To increase from 20 to $28 \mathrm{fps}$, a $2.6 \mathrm{~dB}$ increase in transmit power is required.

An example of the images from Figure 3.5 transmitted over this link at two different bit error rates are shown in Figure 3.8. The received images are shown for BER $=10^{-2}$ and BER $=10^{-3}$. The quality of the $10^{-2}$ BER image is poor as bit errors make it appear that there are more stars in the image than there actually are. But, as the BER starts to go below $10^{-3}$, the images look sufficient. This error rate can be achieved using the QPSK-based system at $10 \mathrm{fps}$, requiring the $E_{s} / N_{0} \geq-0.3 \mathrm{~dB}$, with log-MAP decoding. 


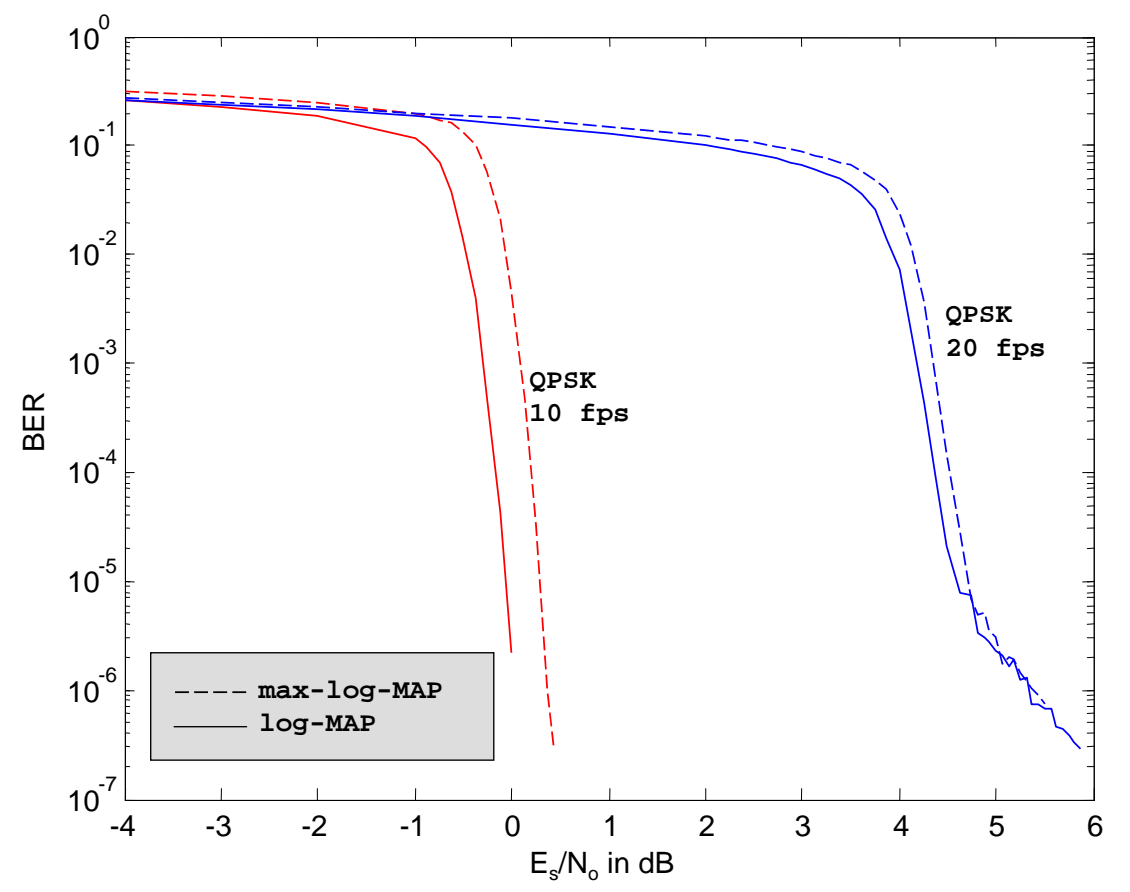

Figure 3.6: Bit Error Performance of two QPSK-based systems in AWGN

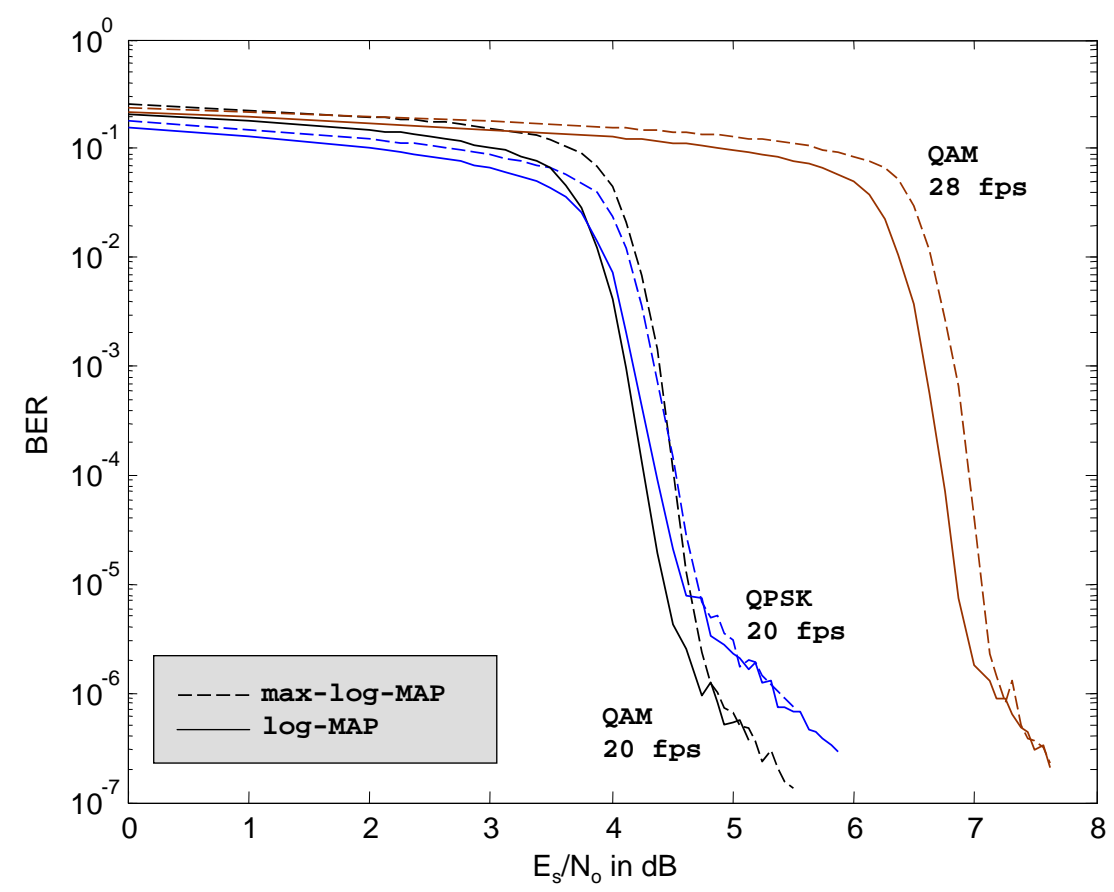

Figure 3.7: Bit Error Performance of one QPSK-based and two QAM-based systems in AWGN 


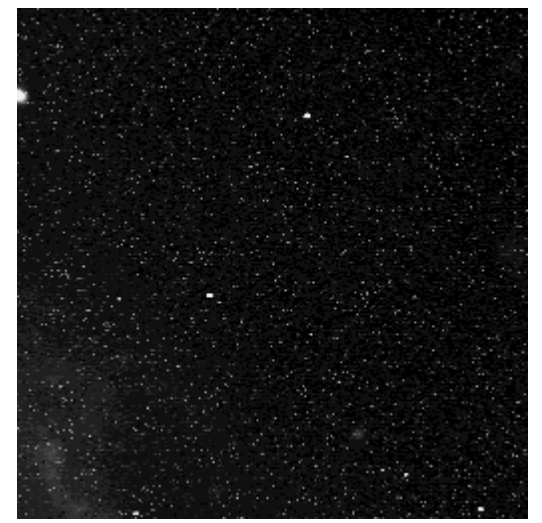

(a) Error Rate $=10^{-2}$

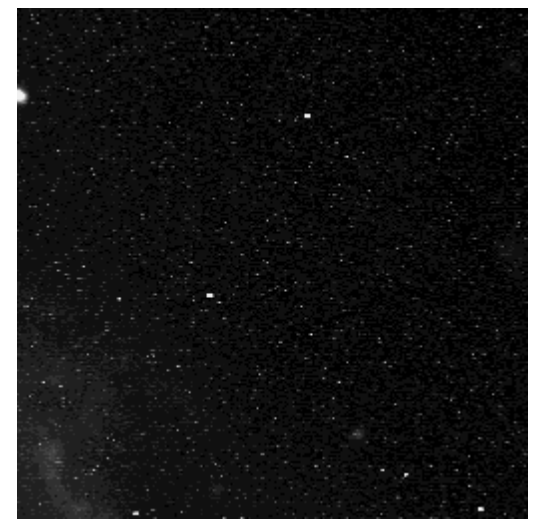

(b) Error Rate $=10^{-3}$

Figure 3.8: Comparison of received images at different error rates

\subsection{Image Processing}

When the images are decoded at the relay, they are then compressed. Through experimentation, images such as the one in Figure 3.5, are easily compacted (lossless compression), using the Lempel-Ziv-Welch (LZW) compaction algorithm. The Tagged image File Format (TIFF) is capable of this kind of compaction, while the Joint Photographic Experts Group (JPEG) uses lossy compression. By compressing the image in Figure 3.5 into a TIFF file with LZW compaction, the file is reduced in size from 32,768 bytes to 8,722 bytes, a $73 \%$ reduction in size. Because the compression is lossless the new image looks exactly the same as that in Figure 3.5.

The effectiveness of the TIFF format with LZW compaction is very dependent on the number of bits of resolution in the original image. In our original image we only used 4 bits of resolution, but the same image with 8 bits of resolution is used the file size is only reduced from 65,536 bytes to 47,750 bytes, which is only a $27 \%$ reduction in size. The data rate would have to increase by a factor of $>5$ in order to double the number of bits of resolution.

The amount of compression that can be performed also depends on the error rate from the source to relay, since errors get compressed too. If there are errors over the link from source to relay, the compressed image is likely to not be compressable to $27 \%$ of its original 
size. For instance, when BER is $10^{-2}$ as in Figure 3.8(a), the compressed image is 26,820 bytes, while if the BER is $10^{-3}$ as in Figure 3.8(a), the compressed image is 19,414 bytes. So when there are errors in the link, there isn't as much to gain from the compaction of LZW. In this thesis, it is assumed that the average compressed image size is 20 kbytes, which allows for non-negligible bit error rate in the link from source to relay. 


\section{Chapter 4}

\section{Information-Outage Analysis}

\subsection{Information-Outage}

In order to analyze the link from the source to the relay, only eight simulations need to be run (4 link types and 2 decoding algorithms), but in order to analyze the link from relay to destination many more simulations will need to be run. The reasons for the extra simulations are because the link from relay to destination will use HARQ, causing each codeword to be decoded up to four times. The channel for this link is also frequency-selective and time correlated, so simulations for different channel conditions will need to be performed. There can also be up to three antennas on the ground each using a rake receiver with up to four fingers. This link will be discussed in Chapter 5

The use of information-outage probability (IOP) as an approximation to the frame error rate of using the actual turbo code, is proposed in order to speed up simulation results. Information-outage probability is defined as the probability that the mutual information falls below the transmission rate [19].

Figure 4.1 shows the IOP concept. The turbo decoder shown in this figure generates a codeword vector $\mathbf{c}$ of length $n$ by taking the length $k$ message vector and passing through the rate $R=k / n$ turbo decoder. This vector is then modulated to produce a length $\frac{n}{\log _{2} M}$ symbol vector $\mathbf{x}$, where $M$ is the size of the signal set (four for QPSK and 16 for 16-QAM). The modulated codeword is sent through the channel (AWGN or Fadinig), which produces the output $\mathbf{y}$. This vector $\mathbf{y}$ is then passed through a demodulator that determines the log 


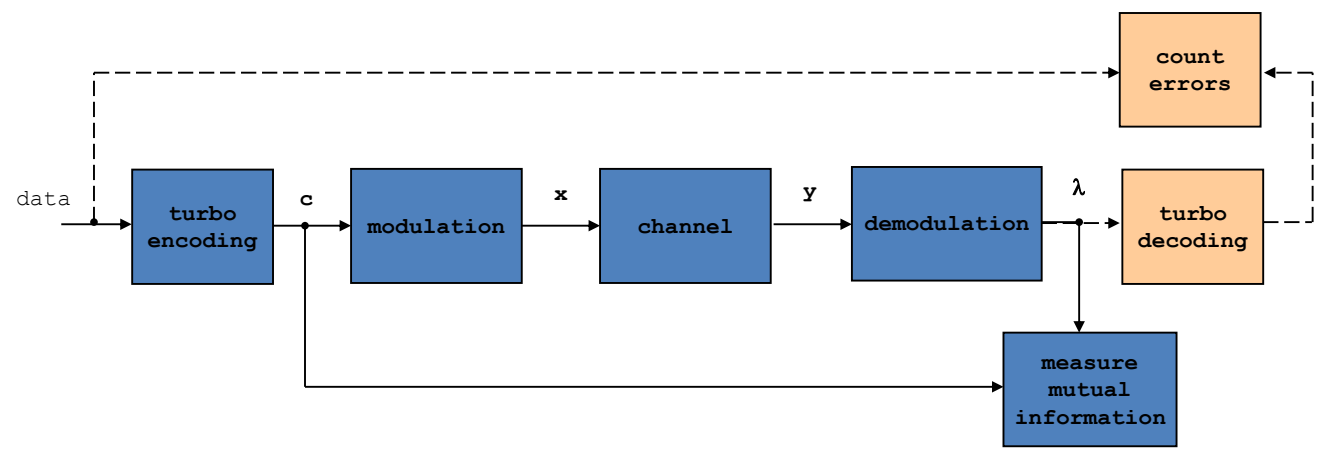

Figure 4.1: Information-Outage Probability Concept

likelihood ratio (LLR) $\boldsymbol{\lambda}$ by determining the LLR $\lambda_{j}$ of each code bit. The LLR of the $j^{\text {th }}$ code bit is calculated using [20]

$$
\lambda_{j}=\log \frac{\operatorname{Pr}\left[c_{j}=1\right]}{\operatorname{Pr}\left[c_{j}=0\right]}=\log \frac{\sum_{x^{\prime} \in \mathcal{S}_{j}^{(1)}} p\left(y \mid x^{\prime}\right)}{\sum_{x^{\prime} \in \mathcal{S}_{j}^{(0)}} p\left(y \mid x^{\prime}\right)}
$$

were $\mathcal{S}_{j}^{(1)}$ is the set of M/2 symbols in the constellation whose value is 1 in the $j^{\text {th }}$ bit, $\mathcal{S}_{j}^{(0)}$ is the set of $\mathrm{M} / 2$ symbols in the constellation whose value is 0 in the $j^{\text {th }}$ bit, and $p\left(\mathbf{y} \mid \mathbf{x}^{\prime}\right)$ is the probability that signal vector $\mathbf{y}$ is received when symbol $\mathbf{x}^{\prime}$ is sent.

For both types of simulations (IOP and FER), the process described is the same. In Figure 4.1, the simulation resulting in FER is marked by dashed lines. In this type of simulation, the LLR vector $\boldsymbol{\lambda}$ is passed to the turbo decoder which outputs the decoded data bits. These bits and then compared to the data originally sent, and counted in order to produce bit error (BER) and frame error rate (FER) curves.

The simulation that results in IOP curves bypasses this decoder. It passes the LLRs into a unit that measures the average mutual information $\bar{I}$ between the code bits of $\mathbf{c}$ and the LLRs of the individual bits of $\boldsymbol{\lambda}$. The mutual information $I$ between the $j^{\text {th }}$ code bit and its corresponding LLR $\lambda_{i}$, is found by $[20]$

$$
I_{j}=1+\log _{2} p\left(c_{j} \mid \lambda_{j}\right)
$$


where $p\left(c_{j} \mid \lambda_{j}\right)$ is the probability that the code bit is $c_{j}$ given that the LLR is $\lambda_{j}$. Equation 4.2 can be calculated in the log domain using [21]

$$
I_{j}=1+\frac{\max ^{*}\left(0, \lambda_{j}(-1)^{c_{j}}\right)}{\ln (2)}
$$

where the $\max ^{*}(\cdot, \cdot)$ is defined as $[18]$

$$
\begin{aligned}
\max ^{*}(x, y) & =\log \left(e^{x}+e^{y}\right) \\
& =\max (x, y)+\log \left(1+e^{-|y-x|}\right)
\end{aligned}
$$

Once the mutual information is found for each of the $\mathrm{n}$ code bits, the average mutual information $\bar{I}$ for the codeword can be calculated using

$$
\bar{I}=\frac{1}{n} \sum_{j=1}^{n} I_{j}
$$

Since information-outage occurs when the average mutual information is below the code rate $R$, the outage probability is determined by

$$
P_{0}=\operatorname{Pr}[\bar{I} \leq R]
$$

In order to show how well the IOP concept works, simulations comparing IOP and logmap turbo decoded systems were generated for configurations \#1 shown in Table 3.1, \#3 shown in Table 3.3, and \#4 shown in Table 3.4 from Section 3.1. The comparison between the results using IOP and FER are shown in Figure 4.2. All of these simulations were over AWGN channels, and used 14 log-map decoding iterations. The IOP and FER curves agree very closely. For configuration \#1 (QPSK 10fps) the two curves are separated by $0.75 \mathrm{~dB}$ at an error rate of $10^{-2}$, while configurations \#3 and \#4 (16-QAM @ 20 fps and 28 fps, respectively) have a separation of $1.1 \mathrm{~dB}$ at the same error rate. It is also shown that IOP is a good indicator of the steepness of the curve, but doesn't predict the flattening part.

Figure 4.3 shows the speedup in simulation time obtained by using the IOP concept. This figure was generated by simulating configuration \#4 (16-QAM 28 fps), by using IOP, log-MAP decoding, and max-log-MAP decoding, and shows the number of TTIs that can be simulated on a $2.13 \mathrm{GHz}$ Intel processor. The IOP simulation runs 23 and 17 times faster than the log-MAP and max-log-MAP decoding, respectively, at low SNR. The distance 


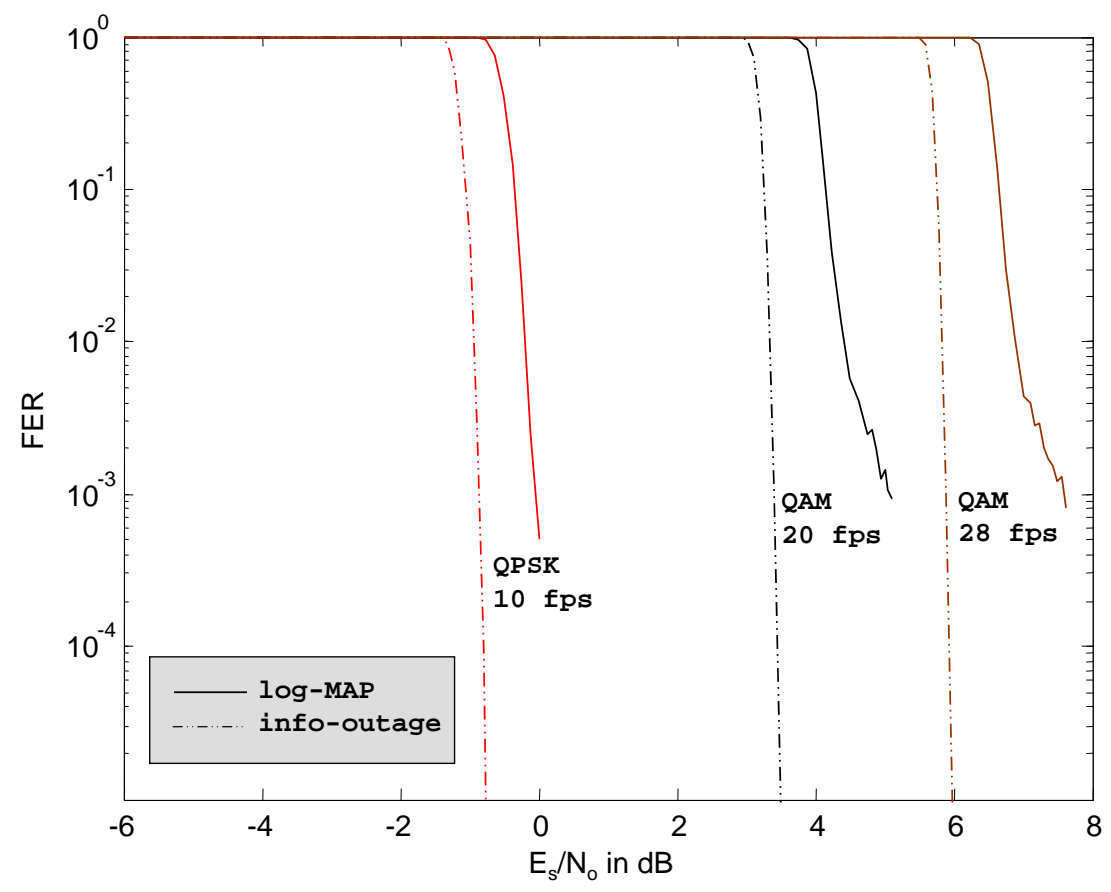

Figure 4.2: Comparison of IOP and FER over three AWGN Channels

decreases at higher SNR due to the decoder not needing all 14 iterations in order to decode the message (stops decoding when CRC check passes).

\subsection{Throughput}

From the FER and IOP curves, throughput of the system at any given signal-to-noise Ratio (SNR) point can be determined. This can be done by calculating the probability of a success for each number of HARQ transmissions, and using the pmf generated to determine the percentage of the maximum throughput transferred through the system. The probability of a success on any given HARQ transmission can be calculated by

$$
\operatorname{Pr}_{S N R}\left[A R Q_{i}\right]=\left(1-\operatorname{FER}_{S N R}\left(A R Q_{i}\right)\right)\left(\prod_{j=1}^{A R Q_{i}-1}\left(\operatorname{FER}_{S N R}(j)\right)\right)
$$




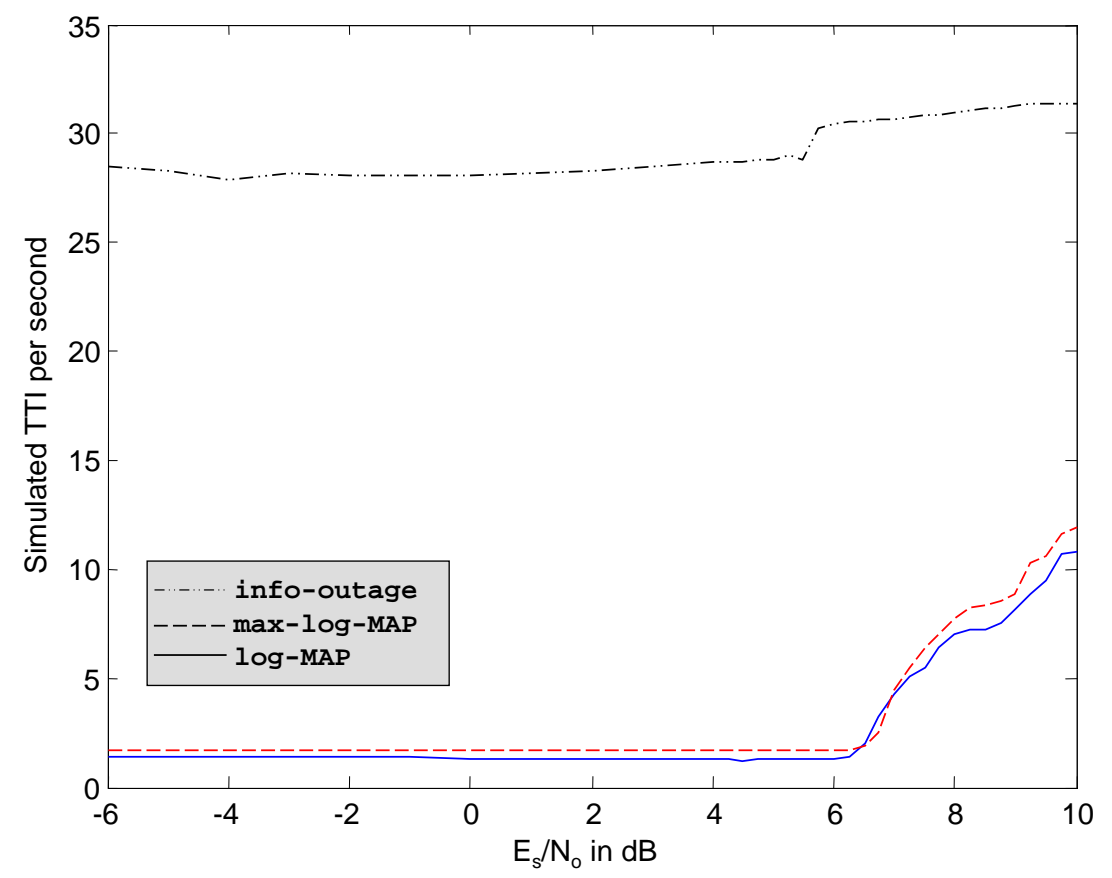

Figure 4.3: Comparison of simulation runtime

if $A R Q_{i}>1$, but by

$$
\operatorname{Pr}_{S N R}\left[A R Q_{i}\right]=1-\operatorname{FER}_{S N R}\left(A R Q_{i}\right)
$$

if $A R Q_{i}=1$, where $A R Q_{i}$ is the number of transmissions before a successful decoding (or no outage), and $\mathrm{FER}_{S N R}\left(A R Q_{i}\right)$ is the frame error rate (or outage probability depending on which simulation is run), where the FER (chance of outage) is equal to chance that HAQR transmission $A R Q_{i}$ is unsuccessful.

Because this system only transmits the message up to four times, the probability mass function (pmf) generated using the above method will not sum to one, so must be normalized 
to make it a valid pmf. This is done by using Equation 4.9

$$
p_{S N R}[A R Q]=\frac{\sum_{j=1}^{A R Q_{\text {Max }}} \operatorname{Pr}_{S N R}\left[A R Q_{j}\right] \delta(A R Q-j)}{\sum_{i=1}^{A R Q_{M a x}} \operatorname{Pr}_{S N R}\left[A R Q_{i}\right]}
$$

where the numerator is the pmf before normalization, $\delta$ is the Dirac delta function, and the denominator is the sum of all the probabilities in the previous pmf.

Once a valid pmf is generated the expected number of ARQ transmissions for a given SNR point can be determined using

$$
E_{S N R}[A R Q]=\sum_{i=1}^{A R Q_{\operatorname{Max}}} p_{S N R}\left[A R Q_{i}\right] i
$$

where $A R Q_{M a x}$ is the maximum number of HARQ transmissions allowed in the system.

Next, the percentage of the maximum throughput on the link can then be calculated

$$
\text { throughput }_{S N R} \%=\frac{1-\mathrm{FER}_{S N R}(M A R Q)}{E_{S N R}[A R Q]}
$$

MARQ is the highest ARQ from the FER Curve for that SNR point plus one except for when the highest is equal to $A R Q_{M a x}$, in which case it is set equal to $A R G_{\text {Max }}$, which in this system is 4 the highest is 4 . In these simulations, the simulation for any given FER (outage) curve is stopped once the minimum FER is reached, so some SNR points will not have results for all numbers of HARQ transmissions. Figures 5.1 and 5.2 show results for a system that will be discussed in Section 5.2. 


\section{Chapter 5}

\section{Relay to Destination Link}

\subsection{Modulation and Coding Schemes}

The link from relay to destination, like the link from source to relay, is based on the HSDPA standard that was described in Section 3.1. This link, though, operates over much longer distances and over frequency-selective fading channels, like that described in Section 2.3.1. In order to counter the effects of the fading channel, HARQ is used on the relay to destination link, which also adds time diversity to the system. If the first transmission cannot be decoded by the destination, up to three retransmissions can be requested. The four total transmissions are encoded slightly differently based on the incremental redundancy (IR) operating mode. In IR mode, a different set of code bits are punctured during each rate-matching process. Also, if 16-QAM modulation is used, the symbol-labeling map is rearranged, which provides signal constellation diversity.

Since the link between relay and destination will have a long round-trip propagation time for the feedback (ACK/NACK), the system will run several processes in parallel such as was shown in Figure 3.4. The reference 25.101 [17] calls for six parallel processes, which would be able to account for time in a system with $1500 \mathrm{~km}$ transmission distance. If the distance would need to be increased, more HARQ processes could be used. The use of parallel HARQ processes adds a high level of time diversity to the system by making the effective duration of a single codeword very long, when retransmissions are taken into account. This also has the added benefit of helping with long signal decorrelation times. 


\begin{tabular}{|l|c|}
\hline \# Physical Channels & 5 \\
\hline Message Bits per TTI & 3202 bits \\
\hline Maximum Data Rate (With no HARQ Retransmissions) & $1.601 \mathrm{Mbps}$ \\
\hline CRC Coded Bit Length & 3226 \\
\hline \# Code Blocks & 1 \\
\hline $\begin{array}{l}\text { Post Encoding and Rate Matching Turbo } \\
\text { Codeword Length (For Each Code Block) }\end{array}$ & $9690 \mathrm{bits}$ \\
\hline Incremental Redundancy Buffer Size & $9600 \mathrm{bits}$ \\
\hline \# of Permanently Punctured Bits & 90 \\
\hline $\begin{array}{l}\text { \# bits selected from IR Buffer } \\
\text { per transmission }\end{array}$ & $4800 \mathrm{bits}$ \\
\hline Code Rate per Transmission $\frac{\text { \# Code Blocks)(\# Bits from IR Buffer })}{\text { Message Bits Per TTT }}$ & 0.6671 \\
\hline
\end{tabular}

Table 5.1: MCS 1 - H-Set 3 QPSK

\begin{tabular}{|l|c|}
\hline \# Physical Channels & 4 \\
\hline Message Bits per TTI & 4664 bits \\
\hline Maximum Data Rate (With no HARQ Retransmissions) & 2.332 Mbps \\
\hline CRC Coded Bit Length & 4688 \\
\hline \# Code Blocks & 1 \\
\hline $\begin{array}{l}\text { Post Encoding and Rate Matching Turbo } \\
\text { Codeword Length (For Each Code Block) }\end{array}$ & $14076 \mathrm{bits}$ \\
\hline Incremental Redundancy Buffer Size & $9600 \mathrm{bits}$ \\
\hline \# of Permanently Punctured Bits & 4476 \\
\hline $\begin{array}{l}\text { \# bits selected from IR Buffer } \\
\text { per transmission }\end{array}$ & $7680 \mathrm{bits}$ \\
\hline Code Rate per Transmission $\frac{\text { Message Bits perTTI }}{(\text { Code Blocks)(\# Bits from IR Buffer) }}$ & 0.6073 \\
\hline
\end{tabular}

Table 5.2: MCS 2 - H-Set 3 16-QAM

In this thesis, four modulation and coding schemes (MCS) are simulated,which are from the 25.101 [17] reference standard. In the 25.101 document they are named H-Set-3-QPSK, H-Set-3-QAM, H-Set-6-QPSK, and H-Set-6-QAM. In this thesis they have been renamed them MCS 1, MCS 2, MCS 3, and MCS 4, respectively. A description of these schemes are found in Tables 5.1, 5.2, 5.3, and 5.4. 


\begin{tabular}{|l|c|}
\hline \# Physical Channels & 10 \\
\hline Message Bits per TTI & 6438 bits \\
\hline Maximum Data Rate (With no HARQ Retransmissions) & 3.219 Mbps \\
\hline CRC Coded Bit Length & 6462 \\
\hline \# Code Blocks & 2 \\
\hline $\begin{array}{l}\text { Post Encoding and Rate Matching Turbo } \\
\text { Codeword Length (For Each Code Block) }\end{array}$ & 9705 bits \\
\hline Incremental Redundancy Buffer Size & 9600 bits \\
\hline \# of Permanently Punctured Bits & 105 \\
\hline $\begin{array}{l}\text { \# bits selected from IR Buffer } \\
\text { per transmission }\end{array}$ & 4800 bits \\
\hline Code Rate per Transmission $\frac{\text { Message Bits per TTI }}{(\# \text { Code Blocks)(\# Bits from IR Buffer })}$ & 0.6706 \\
\hline
\end{tabular}

Table 5.3: MCS 3 - H-Set 6 QPSK

\begin{tabular}{|c|c|}
\hline \# Physical Channels & 8 \\
\hline Message Bits per TTI & 9377 bits \\
\hline Maximum Data Rate (With no HARQ Retransmissions) & $4.689 \mathrm{Mbps}$ \\
\hline CRC Coded Bit Length & 9401 (Padded with 1 bit) \\
\hline \# Code Blocks & 2 \\
\hline $\begin{array}{l}\text { Post Encoding and Rate Matching Turbo } \\
\text { Codeword Length (For Each Code Block) }\end{array}$ & 14115 bits \\
\hline Incremental Redundancy Buffer Size & 9600 bits \\
\hline \# of Permanently Punctured Bits & 4515 \\
\hline $\begin{array}{l}\text { \# bits selected from IR Buffer } \\
\text { per transmission }\end{array}$ & 7680 bits \\
\hline Code Rate per Transmission $\frac{\text { Message Bits per TTT }}{(\# \text { Code Blocks)(\# Bits from IR Buffer) }}$ & 0.6105 \\
\hline
\end{tabular}

Table 5.4: MCS 4 - H-Set 6 QAM 


\subsection{Link Analysis}

An example simulation was run using MCS 1 as described in Table 5.1, using the channel condition parameters $f_{0} / R_{c}=0.1$ (normalized frequency-selective bandwidth), $\tau_{0} / T_{s}=100$ (normalized signal decorrelation time), and receiver configuration $Q=3$ antennas each using a four fingered rake receiver using $14 \log$-MAP demodulation iterations. Since $R_{c}=$ $11(3.84)=42.24 \mathrm{Mchips} / \mathrm{sec}$ (Barker Code Length * Original Chip Rate), and $R_{s}=240$ kbaud, the frequency-selective bandwidth $f_{0}=4.224 \mathrm{MHz}$, and the decorrelation time $\tau_{0}=$ $417 \mu \mathrm{sec}$. Even though the simulator keeps track of the BER, the FER is more relevant to the HARQ system. This is due to the fact that the frame is retransmitted no matter how many bit errors occur.

The FER for each HARQ transmission of MCS 1 over the specified fading channel is illustrated in Figure 5.1. For comparison purposes, the IOP of these links were also simulated using the methods of Section 4.1, and is also shown. As can be seen in the figure, energy efficiency is improved after each successive HARQ transmission, but with diminishing returns. This improvement is due to the fact that the effective code rate is being reduced. IOP is also a good predictor of the actual system performance, usually being between 1 and $2 \mathrm{~dB}$ better than the FER of the actual turbo code.

Since there can be a variable number of HARQ transmissions, the data rate of the system is also variable. One performance metric for the system is throughput, which is the average number of bits per second that is sent over the link. This simulator also keeps track of throughput, but using the methodology described in Section 4.2. Figure 5.2, shows the throughput (in Mbps) of MCS 1 using the same channel parameters used to create Figure 5.1. Throughput can be determined by simulating either the FER or IOP, where a retransmission is performed whenever there is an information-outage. Using the IOP simulation is shown again to be a good predictor of the actual turbo decoded results.

In order to analyze performance on all four MCS schemes over different channel conditions and receiver configurations, many simulations had to be run. The different parameters that were used in these simulations are:

- MCS Scheme : $1,2,3$, and 4 


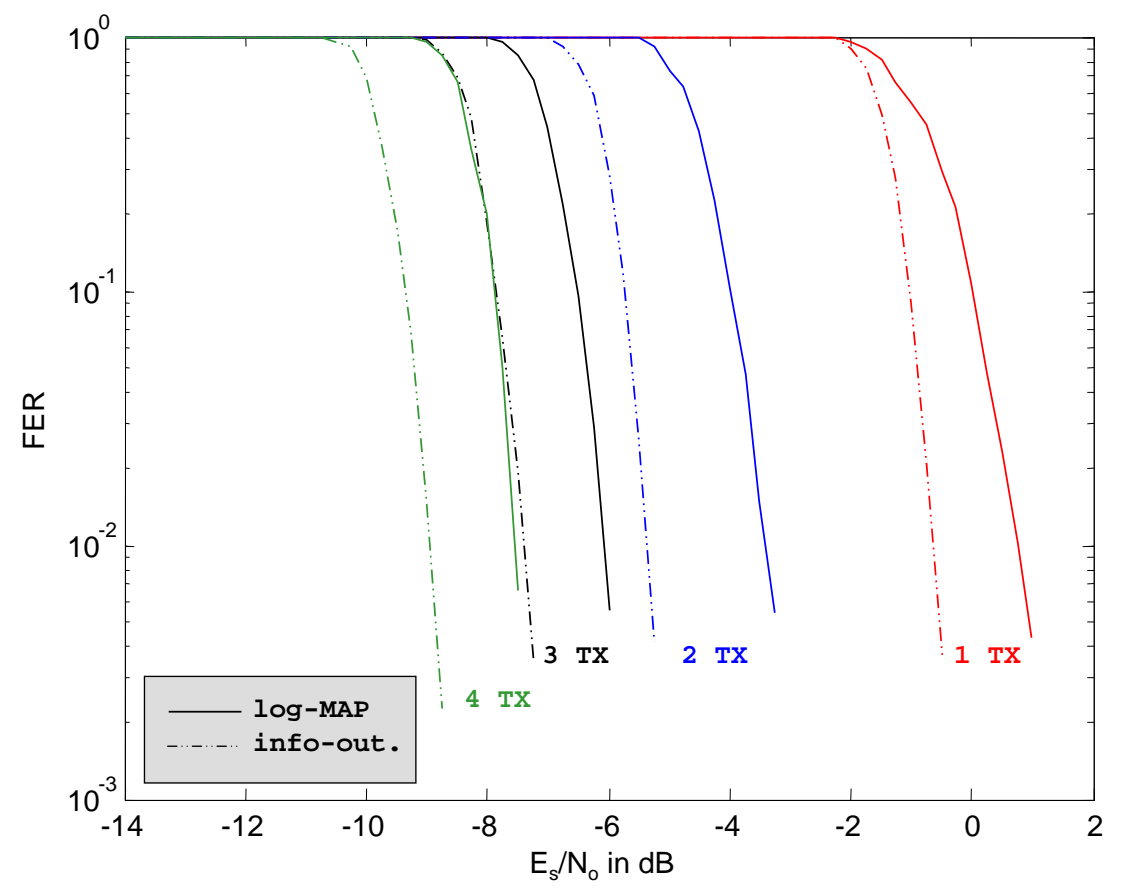

Figure 5.1: Frame Error Rate and Information-Outage Probability Rate for MCS 1

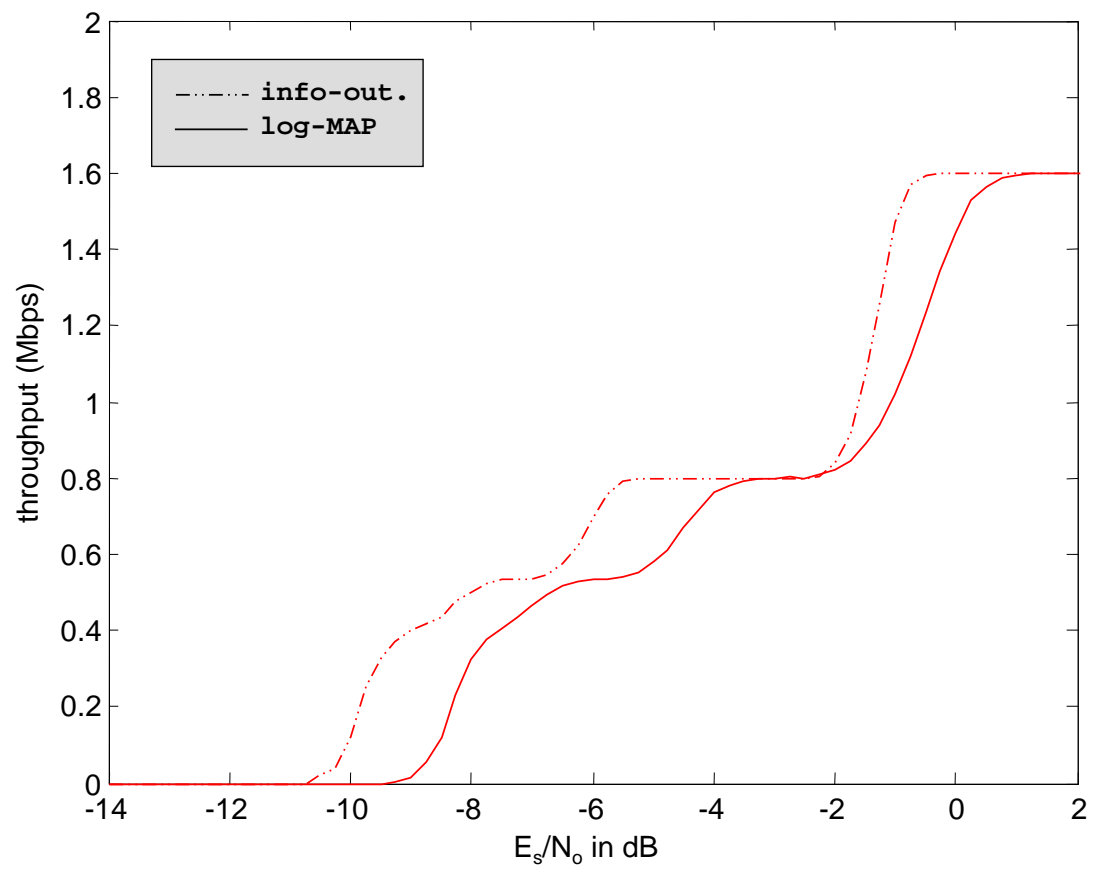

Figure 5.2: Throughput of MCS 1 
- \# of Rake Fingers: 1, 2, 3, and 4

- \# of Receive Antennas: 1, 2, and 3

- Normalized Signal Decorrelation Times: $\tau_{0} / T_{s}=100,200$, and 400

- Normalized Frequency-Selective Bandwidths: $f_{0} / R_{c}=0.05,0.1$, and 0.2

This list requires that 432 simulations are run, and since the runtime of simulations using the turbo decoder run much slower it would be time prohibitive. Since the IOP simulations are a good predictor of the FER simulation results and run much faster, IOP is used to generate initial results. Once the initial results are generated, certain designs and be chosen to be simulated using the turbo decoder in order to get more accurate results.

The 431 simulations were run using the IOP concept from Section 4.1, over two months on eight computers. The main results of these simulations are shown on Figures 5.3, 5.4, 5.5, and 5.6. These figures show the expected frame rate of the video as a function of $\operatorname{SNR}\left(E_{s} / N_{0}\right)$, which is calculated by dividing the throughput by 160,000 ( 8 bits/byte $* 20$ kbytes/frame), since our average image size was assumed to be 20 kbytes.

The influence of the number of rake receiver fingers and the number of antennas is shown in Figure 5.3. This simulation was run using MCS 1, with channel parameters normalized frequency-selective bandwidth $f_{0} / R_{c}=0.1$ and a normalized signal decorrelation time $\tau_{0} / T_{s}=100$. The rake receiver was compared to have between one and four fingers, on either one or three receive antennas. As can be seen in Figure 5.3, there is a $6 \mathrm{~dB}$ gain for using four fingers over one when using one antenna, and a $4 \mathrm{~dB}$ gain when using three antennas. Also, when four fingers are used, there is a $5.5 \mathrm{~dB}$ gain when using three antennas over one.

The throughput of the four different MCS types are shown in Figure 5.4. The channel configuration used for this simulation was a frequency-selective bandwidth $f_{0} / R_{c}=0.1$, and normalized signal decorrelation time $\tau_{0} / T_{s}=100$, with receiver configuration $L=4$ fingered rake receiver, and $Q=3$ antennas. As can be seen in the Figure, MCS 3 and 4 are generally more efficient than MCS 1 and 2, which also is seen in other channel conditions. The efficiency increase can be attributed to MCSs 3 and 4 use of double the physical channels as MCS 1 and 2, respectively. From this observation it is preferable to simulate MCSs 3 and 


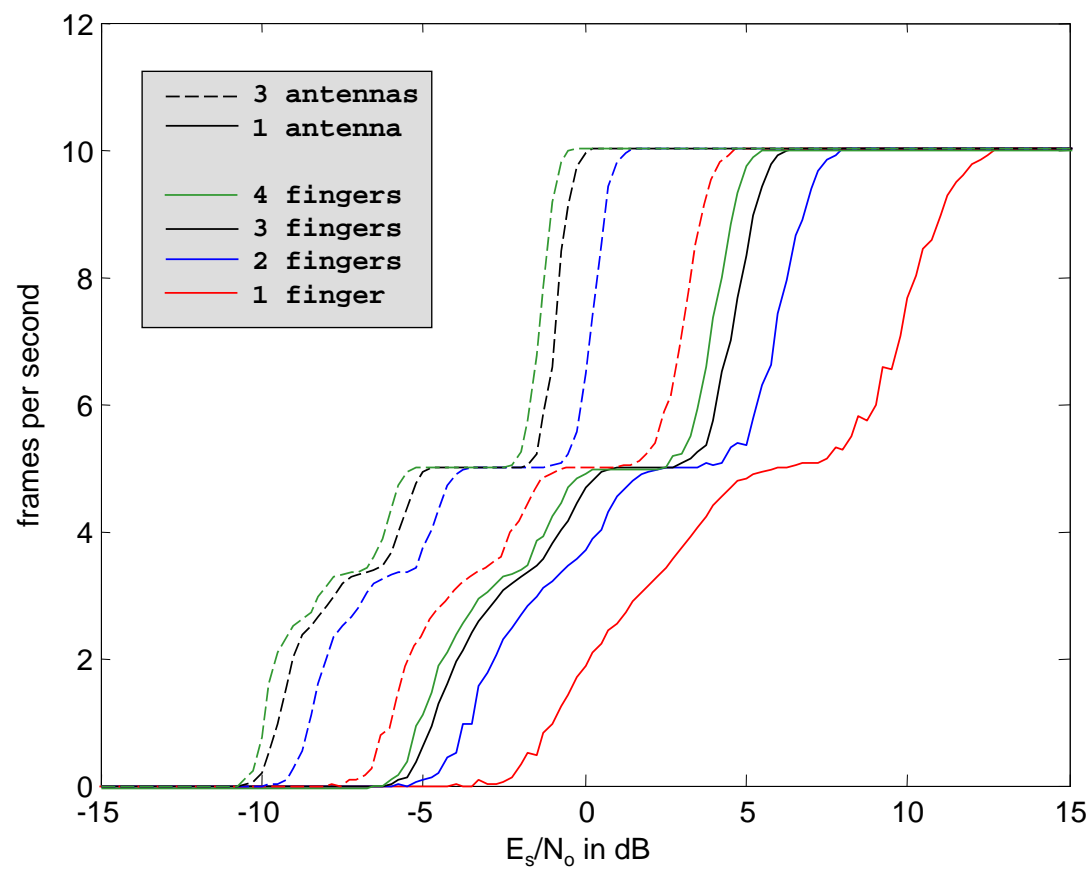

Figure 5.3: Influence of the amount of rake fingers and number of antennas on MCS 1

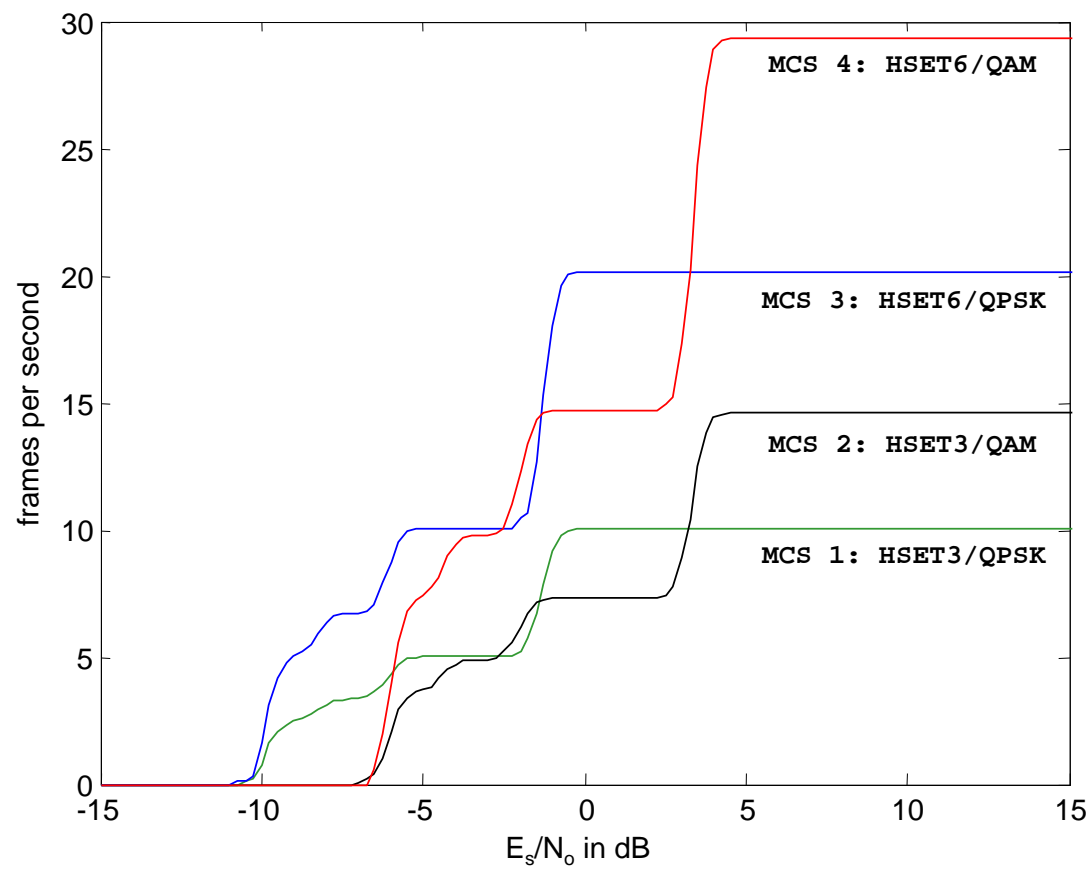

Figure 5.4: Throughput of all MCS schemes over the a fading channel 


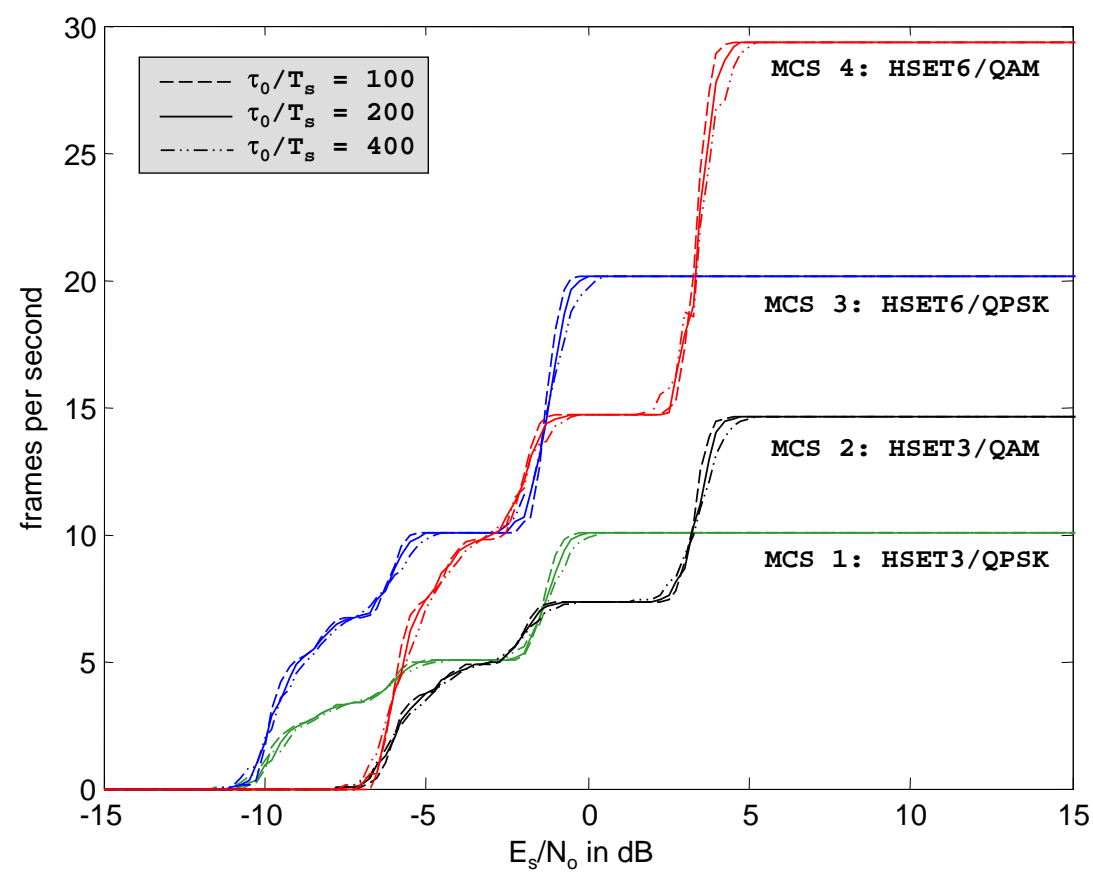

Figure 5.5: Influence of Signal Decorrelation Time on System

4 over MCSs 1 and 2 without even performing the turbo decoding simulation. Also, from this figure we can see that at low SNR MCS 3 is more efficient than MCS 4, except for a small region $-3<E_{s} / N_{0}<-1 \mathrm{~dB}$, while MCS 4 is preferable for $E_{s} / N_{0}>3.5 d B$.

Figure 5.5 shows how the normalized signal decorrelation time affects the system. For this system, the channel condition held constant was the normalized frequency-selective bandwidth of $f_{0} / R_{c}=0.1$, using $L=4$ fingers for each rake receiver, and $Q=3$ antennas, while the normalized signal decorrelation time was varied. The results for all four MCS systems are shown for normalized decorrelation times $\tau_{0} / T_{s}=\{100,200,400\}$. With a normalized decorrelation time of 400, the longest absolute decorrelation time $\tau_{0}$ will be $1.67 \mathrm{msec}$, almost an entire TTI $(2 \mathrm{msec})$. Even though the channel can produce longer decorrelation times, the effect of these longer times will be reduced by using multiple HARQ processes as in Figure 3.4. In Figure 3.4, the system uses 6 processes interleaved in a round- 


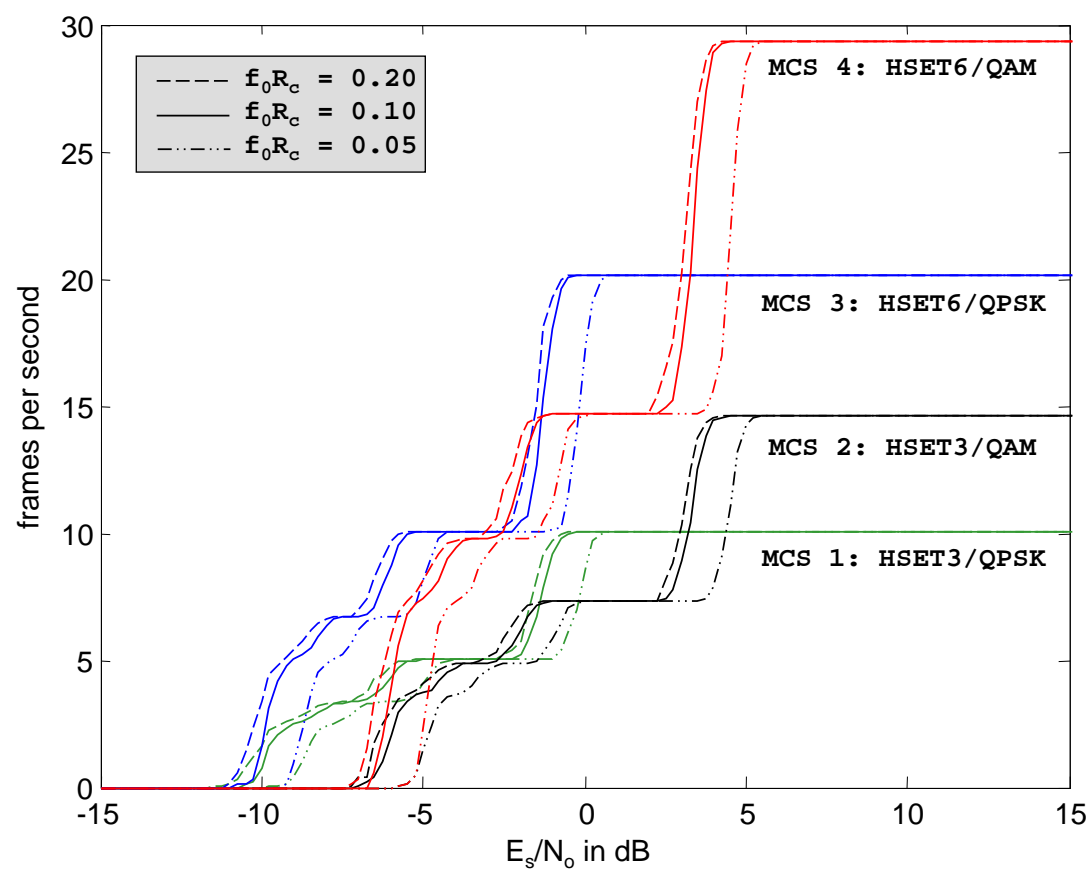

Figure 5.6: Influence of Frequency-Selective Bandwidth on System

robin, allowing the system to handle signal decorrelation times on the order of $10 \mathrm{msec}$. Longer decorrelation times can be resisted even more by adding other HARQ processes, but this will increase complexity and the latency of the system.

Figure 5.6 shows how the normalized frequency-selective bandwidth affects the system. For this system, the channel condition parameter held constant was the normalized signal decorrelation time $\tau_{0} / T_{s}=100$, using $L=4$ fingers for each rake receiver, and $Q=3$ antennas, while the normalized frequency-selective bandwidth was varied. The results for all four MCS systems are shown for normalized decorrelation times $f_{0} / R_{c}=\{0.05,0.1,0.2\}$. This figure shows that as $f_{0} / R_{c}$ is increased, so is performance. The gain from increasing $f_{0} / R_{c}$ from 0.05 to 0.1 is about $2 \mathrm{~dB}$, while the increase from 0.1 to 0.2 is about $0.5 \mathrm{~dB}$. The large gain, from $f_{0} / R_{c}=0.05$ to $f_{0} / R_{c}=0.1$, is due to the channel becoming less dispersive as the frequency-selective bandwidth becomes larger. As can be seen from Equation 2.33 


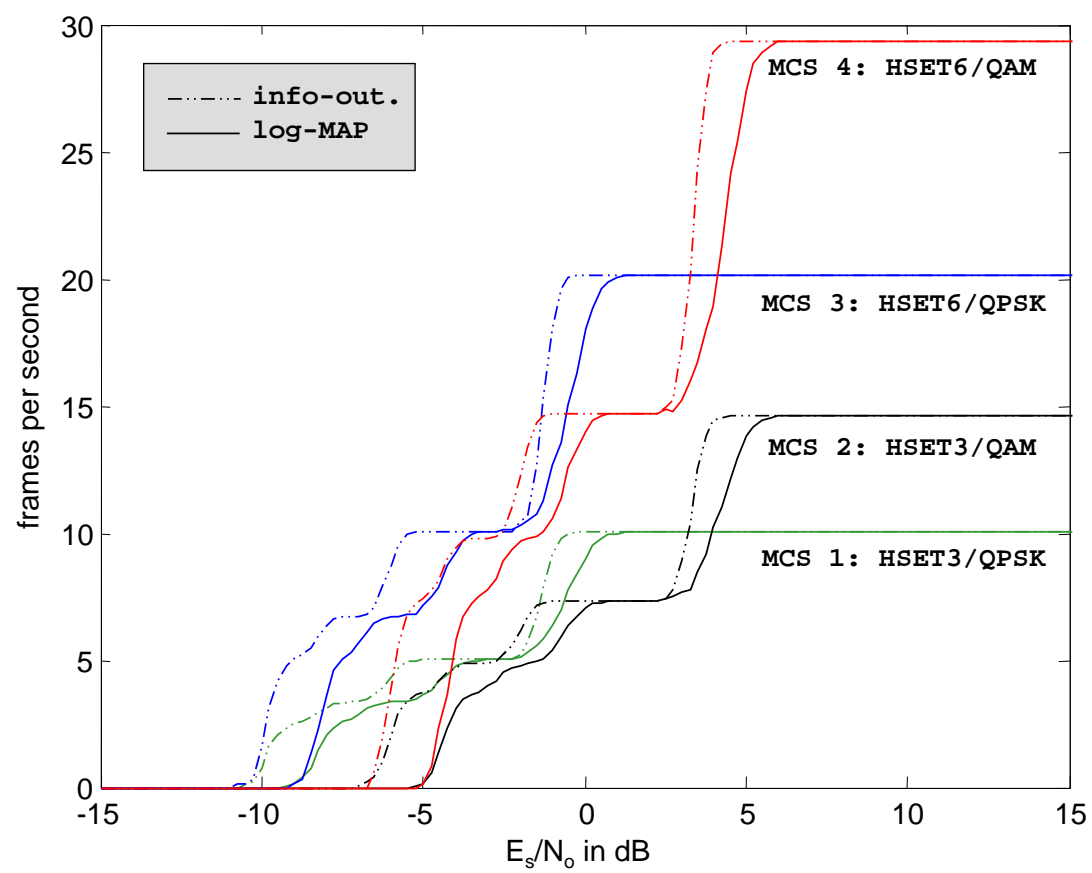

Figure 5.7: Comparison of IOP and log-MAP Decoded system throughputs

in Section 2.3.1, the number of taps $K=16$ in a half chip spaced delay line model when $f_{0} / R_{c}=0.1$, while $K=31$ when $f_{0} / R_{c}=0.05$. When using a four fingered rake receiver, most of the energy in the $f_{0} / R_{c}=0.1$ case can be captured, while a significant amount of the energy in the $f_{0} / R_{c}=0.05$ case is received after delay $\tau=3 T_{c}$, making it not obtainable by the rake receiver. More fingers would always be used to counter for narrower frequencyselective bandwidths, but may required a longer spreading code.

Figures 5.3 to 5.6 show the different effects that the conditions of the channel (signal deccorelation time, and frequency-selective bandwidth), and receiver configurations (\# of antennas, \# fingers on rake receiver, MCS scheme) have on the system. These results were generated using the IOP concept, but simulations of actual turbo decoding should be performed once sets of parameters are identified.

A comparison of the throughput (in fps), using IOP and actual turbo coding with 14 
iterations of log-MAP decoding, is shown in Figure 5.7. Curves were generated for all four MCS schemes, with normalized frequency-selectivity $f_{0} / R_{c}=0.1$, normalized decorrelation time $\tau_{0} / T_{s}=100, L=4$ fingered rake receiver, and $Q=3$ antennas. The performance of the IOP simulation is shown to be approximately 1 to $2 \mathrm{~dB}$ better than that simulated by the turbo code.

For the actual turbo decoded system MCS 3 is the best system for all SNRs lower than $E_{s} / N_{0}=4 \mathrm{~dB}$, which differs from the observation of only the IOP curves as discussed previously. This shows that while information-outage is a good predictor of performance, it is important to simulate the actual turbo decoded system. 


\section{Chapter 6}

\section{Conclusions and Future Work}

\subsection{Conclusions}

From the previous simulation results, recommendations for link configurations can be made. First, it is suggested that MCS 3 be used for the relay to destination link, as it appears to be the best link for 20 fps or less according to Figure 5.7. Since the link from relay to destination will be limited to $20 \mathrm{fps}$, the need for a faster link from source to relay is unnecessary. It is recommended to use link 3 (20 fps using 16-QAM) when conditions are well enough, and link 1 (10fps using QPSK) when conditions are poorer.

This proposed configuration for link and receiver appears to be resistant to many conditions on the relay to destination link. The use of H-Set 6 (6 interleaved HARQ processes, with 4 possible transmissions) allows for a maximum decorrelation time of about (4)(6)(2 $\mathrm{msec})=48 \mathrm{msec}(\max \#$ transmissions)(\# interleaved processes)(TTI duration). If the number of interleaved processes are increased this time can be increased. This combined with the secondary spreading of the Barker code, four-finger rake receiver, and three destination antennas allows a frequency-selective bandwidth of approximately one-tenth of the signal bandwidth to be handled. Narrower bandwidths can be handled using more fingers on the rake receiver, but the number of fingers is limited by the length of the secondary spreading code (11 due to use of the Barker-11 code). A longer spreading code could also be used, which would enable the increase in the maximum number of fingers on the receiver. 


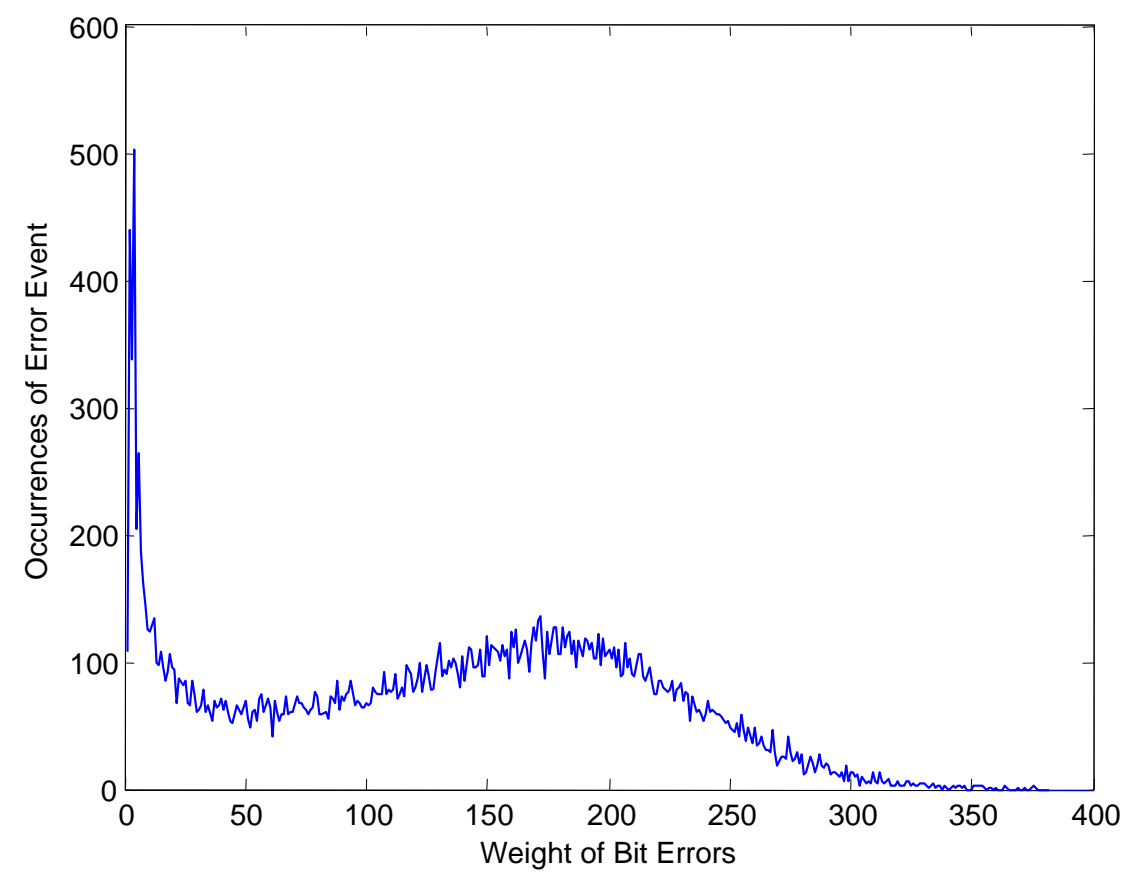

Figure 6.1: Histogram of Bit Errors for 1 million turbo codewords over AWGN channel

\subsection{Suggestions for Future Work}

As can be seen in Figure 1.1, some of the original signal sent from source to relay will end up at the destination. This signal will be very weak in comparison to the information sent from the relay to the destination. Some reasons for this are that the original intent of this signal was to travel over a much shorter distance to the relay, which will give it a much larger free space path loss. Also, the antenna on the source is most likely pointed toward the relay making the antennas at the destination off the boresight of the source antennas. Since the antennas at the destination most likely have a higher gain than the antennas on the relay, some of this loss can be compensated for.

Methods of exploiting this extra information, would help to improve the overall performance of the system. One method that may be useful would be to decode the information from the source and relay and perform some type of combining of the data. Also in our 
simulations, when an error did occur the most common number of bit errors were four. A histogram showing the number of errors can be seen in Figure 6.1. The system may then be able to take the lowest four LLRs of the data and flip the bits that correspond to those LLRs, in order to increase performance. This process could also be performed at the relay though, and not necessarily at the destination. 


\section{References}

[1] Andrea Goldmsmith, Wireless Communications, Cambridge University Press, New York, NY, first edition, 2005.

[2] T. M. Cover and A. A. El Gamal, "Capacity theorems for the relay channel," IEEE Trans. Inform. Theory, vol. 25, pp. 572-584, Sept. 1979.

[3] R. Knopp and P. A. Humblet, "On coding for block fading channels," IEEE Trans. Inform. Theory, vol. 46, no. 1, pp. 189-205, Jan. 2000.

[4] P.A. Anghel and M. Kaveh, "Relay assisted uplink communication over frequencyselective channels," Signal Processing Advances in Wireless Communications, 2003. SPAWC 2003. 4th IEEE Workshop on, pp. 125-129, 15-18 June 2003.

[5] J.N. Laneman, D.N.C. Tse, and G.W. Wornell, "Cooperative diversity in wireless networks: Efficient protocols and outage behavior," Information Theory, IEEE Transactions on, vol. 50, no. 12, pp. 3062-3080, Dec. 2004.

[6] A. Sendonaris, E. Erkip, and B. Aazhang, "User cooperation diversity. part i. system description," Communications, IEEE Transactions on, vol. 51, no. 11, pp. 1927-1938, Nov. 2003.

[7] Bin Zhao and M.C. Valenti, "Practical relay networks: a generalization of hybrid-arq," Selected Areas in Communications, IEEE Journal on, vol. 23, no. 1, pp. 7-18, Jan. 2005.

[8] Robert L. Bogusch, "Digital communications in fading channels: Modulation and coding," Tech. Rep., Mission Research Corporation, Report for AFWL, AFWL-TR-87-52, April 1989.

[9] Roger A. Dana, "Statistics of samples rician fading," Tech. Rep., Mission Research Corporation, DNA-TR-92-98, (Revised)March 2000.

[10] J. Proakis, Digital Communications, McGraw-Hill, Inc., New York, NY, fourth edition, 2001.

[11] R.H. Clarke, "A statistical theory of mobile-radio reception," in Bell Systems Technical Journal, pp. 957-1000. Bell Systems, July-August 1968.

[12] P. Hoeher, "A statistical discrete-time model for the WSSUS multipath channel," IEEE Trans. Veh. Tech., vol. 41, no. 4, pp. 461-468, May 1992. 
[13] L.W. Couch, Digital and Analog Communication Systems, Prentice Hall, fifth edition, 1997.

[14] European Telecommunications Standards Institute (ETSI), "UTRA (BS) FDD; radio transmission and reception," 3GPP TS 25.104 Version 6.6.0, September 2005.

[15] European Telecommunications Standards Institute (ETSI), "Universal mobile telecommunications system (UMTS): Multiplexing and channel coding (FDD)," 3GPP TS 25.212 Version 6.6.0, September 2005.

[16] European Telecommunications Standards Institute (ETSI), "Universal mobile telecommunications system (UMTS): Spreading and modulation (FDD)," 3GPP TS 25.213 Version 6.4.0, September 2005.

[17] European Telecommunications Standards Institute (ETSI), "Universal mobile telecommunications system (UMTS): User equipment (UE) radio transmission and reception (FDD)," 3GPP TS 25.101 Version 6.9.0, September 2005.

[18] A. J. Viterbi, "An intuitive justification and a simplified implemetation of the MAP decoder for convolutional codes," IEEE Journal on Selected Areas in Communications, vol. 16, no. 2, pp. 260-264, February 1998.

[19] J. N. Laneman, "On the distribution of mutual information," in Proc. Workshop on Information Theory and its Applications, San Diego, CA, February 2006.

[20] G. Caire, G. Taricco, and E. Biglieri, "Bit-interleaved coded modulation," IEEE Trans. Inform. Theory, vol. 44, pp. 927-946, May 1998.

[21] R.I. Seshadri and M.C. Valenti, "Capacity-based parameter optimization of bitinterleaved coded CPM with differential detection," IEEE Trans. Veh. Tech., vol. 57, no. 1, January 2008. 